\title{
MODULES OVER COPRODUCTS OF RINGS
}

\author{
BY
}

\author{
GEORGE M. BERGMAN ( $\left.{ }^{(}\right)$
}

\begin{abstract}
Let $R_{0}$ be a skew field, or more generally, a finite product of full matrix rings over skew fields. Let $\left(R_{\lambda}\right)_{\lambda \in \Lambda}$ be a family of faithful $R_{0^{-}}$ rings (associative unitary rings containing $R_{0}$ ) and let $R$ denote the coproduct ("free product") of the $R_{\lambda}$ as $R_{0}$-rings. An easy way to obtain an $R$-module $M$ is to choose for each $\lambda \in \Lambda \cup\{0\}$ an $R_{\lambda}$-module $M_{\lambda}$, and put $M=$ $\bigoplus_{M_{\lambda}} \otimes_{R_{\lambda}} R$. Such an $M$ will be called a "standard" $R$-module. (Note that these include the free $R$-modules.)

We obtain results on the structure of standard $R$-modules and homomorphisms between them, and hence on the homological properties of $R$. In particular:
\end{abstract}

(1) Every submodule of a standard module is isomorphic to a standard module.

(2) If $M$ and $N$ are standard modules, we obtain simple criteria, in terms of the original modules $M_{\lambda}, N_{\lambda}$, for $N$ to be a homomorphic image of $M$, respectively isomorphic to a direct summand of $M$, respectively isomornhic to $M$.

(3) We find that $\mathrm{r} g l \operatorname{dim} R=\sup _{\Lambda}\left(\mathrm{rgl} \operatorname{dim} R_{\lambda}\right)$ if this is $>0$, and is 0 or 1 in the remaining case.

In $\$ 2$ below we shall state our main results, Theorems 2.2 and 2.3 , and derive a large number of consequences. In $\S \S 3-8$ we prove these theorems, assuming, to avoid distractions, that $R_{0}$ is a skew field. Afterward $(\S \S 9,10)$ we indicate how to adapt the proofs to the case of $R_{0}$ a finite direct product of full matrix rings over skew fields, examine some simple examples $(\S 12)$, and discuss possible generalizations of our results ( $\S 11,13-15)$.

Some important applications of these results will be made in [3] .

The idea of our proofs goes back to P. M. Cohn's work [8], [9], [14] . I

Received by the editors October 15, 1973.

AMS (MOS) subject classifications (1970). Primary 16A06, 16A54, 16A64, 18F25; Secondary 08A10, 16A40, 16A48, 16A50, 16A54, 16A60, 16A62, 18A30, 18C10, 18E05, $20 \mathrm{C05}$.

Key words and phrases. Coproduct (or "free product") of rings, category of $R$-modules, global dimension.

(1) These results were obtained while the author held an AFOSR Postdoctoral Fellowship and written up while the author was an Alfred P. Sloan Fellow. During the preparation of the final revision, the author was supported by NSF contract GP37492X. 
am grateful to P. M. Cohn, A. J. Czerniakiewicz, and R. Gordon for helpful criticisms of an earlier draft of this paper.

1. Definitions, conventions, and some basic tools. All rings will be associative and have 1, all homomorphisms and modules will be assumed unital, and module will mean right module unless the contrary is stated. Sfield means skew field (division ring).

Given a ring $R_{0}$, an $R_{0}$-ring means a ring $R$ given with a homomorphism $R_{0} \rightarrow R$. An $R_{0}$-ring is called faithful if the given homomorphism is an embedding. The $R_{0}$-rings form a category in which the maps are the ring homomorphisms that form commutative triangles with the given maps from $R_{\mathbf{0}}$.

Throughout what follows, $R_{0}$ will be a fixed ring, and $\left(R_{\lambda}\right)_{\lambda \in \Lambda}$ a family of $R_{0}$-rings. We shall denote by $R$ the coproduct of the $R_{\lambda}$ in the category of $R_{0}$-rings.( ${ }^{2}$ )

We shall generally use the letter $\lambda$ for an index in $\Lambda$, and $\mu$ for an index in $\Lambda \cup\{0\}$; thus $R_{\mu}$ means one of the $R_{\lambda}$, or $R_{0}$.

By a standard $R$-module, we shall mean an $R$-module of the form $N=$ $\bigoplus_{\mu \in \Lambda \cup\{0\}} N_{\mu} \otimes_{R_{\mu}} R$, where each $N_{\mu}$ is an $R_{\mu}$-module. (This definition could, of course, be made over any ring $R$ given with a family of maps $R_{\mu} \rightarrow$ $R$.) This $N$ has the universal property that given any $R$-module $P$, and given for each $\mu$ an $R_{\mu}$-module homomorphism $g_{\mu}: N_{\mu} \rightarrow P$, there will exist a unique extension $g$ :

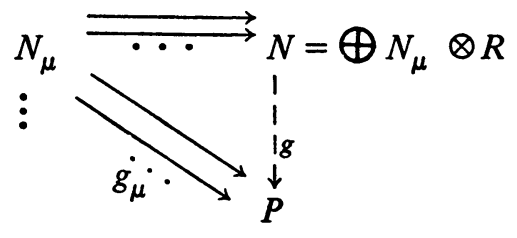

We shall generally abbreviate $\otimes_{R_{\mu}}$ to $\otimes_{\mu}$ or simply $\otimes$, as above.

(2) Equivalently: The fibered coproduct of the $R_{\lambda}$ over $R_{0}$, or the pushout or colimit of the maps $R_{0} \rightarrow R_{\lambda}$ in the category of rings. This exists by general results of universal algebra [13, Theorem III.6.1].

$R$ is also frequently termed "the free product of the $R_{\lambda}$ over $R_{0}$ " (or "with amalgamation of $R_{0}$ ") if the $R_{\lambda}$ are all faithful $R_{0}$-rings, are embedded monomorphically in $R$, and are disjoint in $R$ except for the common image of $R_{0}$. When these conditions hold one says (in this usage) that "the free product $R$ of the $R_{\lambda}$ 's exists". I suggested in $[4, \S 7]$ the alternative statement for the last two of these conditions, "the coproduct $R$ of the $R_{\lambda}$ 's is faithful (over each $R_{\lambda}$ ) and separating".

The term "free product" was adopted from group theory, where coproducts are always faithful and separating. But it goes against modern categorical usage, and we shall speak here of coproducts. 
Under the hypotheses we shall introduce in the next section, we shall find that the canonical maps $R_{\mu} \rightarrow R$ and $N_{\mu} \rightarrow N$ ( $N$ as above) are injections (Proposition 2.1). Hence we may identify $R_{\mu}$ and $N_{\mu}$ with their images in $R$ and $N$. In particular, a standard module can be thought of as an $R$-module $N$ with a distinguished family of $R_{\mu}$-submodules $N_{\mu}$, having the above mapping property; i.e., generating $N$ "freely".

When we speak of a homomorphism (isomorphism, etc.) of standard $R$ modules, we shall simply mean a homomorphism (etc.) as $R$-modules. But there are certain special classes of homomorphisms that we shall define in terms of the standard-module structure.

A homomorphism $f: \bigoplus M_{\mu} \otimes R \rightarrow \bigoplus N_{\mu} \otimes R$ will be called induced if it arises from a family of homomorphisms $f_{\mu}: M_{\mu} \rightarrow N_{\mu}$. Under the injectiveness conditions mentioned above, we see that $f$ is induced if and only if for each $\mu$ it carries $M_{\mu} \subseteq M$ into $N_{\mu} \subseteq N$.

Another type of map arises from the fact that a free $R$-module can be written as a standard module in more than one natural way. Suppose we have a standard module $\bigoplus M_{\mu} \otimes R$, and for some $\mu_{1}$, a decomposition $M_{\mu_{1}} \cong$ $M_{\mu_{1}}^{\prime} \oplus R_{\mu_{1}}$. Then $M_{\mu_{1}} \otimes R \cong\left(M_{\mu_{1}}^{\prime} \otimes R\right) \oplus R$. Hence if for some $\mu_{2} \neq$ $\mu_{1}$, we set $M_{\mu_{2}}^{\prime}=M_{\mu_{2}} \oplus R_{\mu_{2}}$, and for all $\mu \neq \mu_{1}, \mu_{2}$, write $M_{\mu}^{\prime}=M_{\mu}$, we get a natural isomorphism: $\bigoplus M_{\mu} \otimes R \cong \bigoplus M_{\mu}^{\prime} \otimes R$. We shall call an isomorphism of this sort a "free transfer isomorphism". From the point of view that a standard module is an $R$-module $M$ with a certain type of generating family of $R_{\mu}$-submodules, a free transfer isomorphism can be thought of as leaving the module $M$ unchanged, but removing from one $M_{\mu_{1}}$ a free summand $x R_{\mu_{1}}(x \in M)$ and attaching $x R_{\mu_{2}}$ to another $M_{\mu_{2}}$.

Actually, the free transfer maps will be satisfactory for our purposes only when $R_{0}$ is a sfield, so that all $R_{0}$-modules are free. More generally, let us define a basic $R_{\mu}$-module as one of the form $A \otimes_{0} R_{\mu}$, where $A$ is a module over our base-ring $R_{0}$. Clearly, the above discussion of free-transfer isomorphisms extends to "basic-transfer" isomorphisms, which remove from some $M_{\mu_{1}}$ a direct summand $A \otimes_{0} R_{\mu_{1}}$ and transfer it to some $M_{\mu_{2}}$ as $A \otimes_{0} R_{\mu_{2}}$. Note also that when $R_{\mathbf{0}}$ is a finite direct product of full matrix rings over sfields, all $R_{0}$-modules are projective, hence all basic $R_{\mu}$-modules are projective over $R_{\mu}$.

Finally, there is a certain type of automorphism of a standard module $M=\bigoplus M_{\mu} \otimes R$ that we shall need. Suppose that for some $\mu_{1}$, we have an $R_{\mu_{1}}$-linear functional $e: M_{\mu_{1}} \rightarrow R_{\mu_{1}}$, and let us extend it to an $R$-linear functional $\epsilon: M \rightarrow R$ so as to annihilate all $M_{\mu}$ with $\mu \neq \mu_{1}$. Suppose $a$ is any element of $R$, and $\delta: R \rightarrow R$ the map left-multiplication by $a$. 
Finally, for some $\mu_{2}$, let $x \in M_{\mu_{2}}$, and let $\gamma: R \rightarrow M$ take 1 to $x \in$ $M_{\mu_{2}} \otimes R \subseteq M$.

Note that if $\mu_{2} \neq \mu_{1}, \epsilon \gamma=0$. If $\mu_{1}=\mu_{2}$, let us insure this by adding the condition that $x$ lie in the kernel of $e$. Since $\epsilon \gamma$ is now zero (in either case), the map $\gamma \delta \epsilon: M \rightarrow M$ will be nilpotent. Hence $\theta=\mathrm{id}_{M}-\gamma \delta \epsilon$ is an automorphism of $M$. We shall call such a $\theta$ a transvection.

2. Statements of the main theorems, and consequences. Thoughout this section the base-ring $R_{0}$ will be assumed to be a finite direct product of full matrix rings over sfields; that is, a ring of global dimension zero, and the $R_{\lambda}$ will all be assumed faithful $R_{0}$-rings. $R$ will denote their coproduct, as above.

An elementary result we shall obtain in $\S 4$ is:

Proposition 2.1. If $M=\bigoplus M_{\mu} \otimes R$ is a standard $R$-module, then for each $\mu$, (1) the canonical map $M_{\mu} \rightarrow M$ is an embedding, and (2) as an $R_{\mu}$-module, $M$ is the direct sum of the image of this map and a basic (hence projective) $R_{\mu}$-module.

In particular, each $R_{\mu}$ embeds in $R=R_{\mu} \otimes R$, and $R$ is projective as a right (and hence by symmetry of our hypothesis, as a left) $R_{\mu}$-module.

Hence we shall make the natural identifications $R_{\mu} \subseteq R, M_{\mu} \subseteq M$. We now state our main results:

THEOREM 2.2. Any submodule of a standard R-module can be given a structure of standard R-module.

THEOREM 2.3. Let $f: M \rightarrow N$ be a surjective homomorphism of finitely generated standard R-modules, $M=\bigoplus_{M_{\mu}} \otimes R, N=\bigoplus N_{\mu} \otimes R$. Then there exists an isomorphism of standard $R$-modules $\alpha: M^{\prime} \cong M$, which is a finite composition of basic-transfer isomorphisms and transvections, such that $f \alpha$ : $M^{\prime} \rightarrow N$ is an induced homomorphism (i.e., $f \alpha\left(M_{\mu}^{\prime}\right)=N_{\mu}$ for all $\mu$ ).

Or, to put the conclusion of Theorem 2.3 another way, keeping the same $M$, one can modify its structure of standard module (distinguished family of $R_{\mu}$-submodules) by a finite sequence of basic transfers, and transvection automorphisms of $M$, so that under the new structure $f$ is an induced homomorphism.

(In $\S 11$ we shall see that the class of finitely generated modules can be replaced, in Theorem 2.3 and hence in some of the Corollaries, by a wider class.)

Proposition 2.1 gives us:

Corollary 2.4. If $M=\bigoplus M_{\mu} \otimes R$, then $\mathrm{hd}_{R} M=\sup _{\mu}\left(\mathrm{hd}_{R_{\mu}} M_{\mu}\right)$. 
Proof. Clearly, this reduces to showing, for each $\mu$, that $\mathrm{hd}_{R} M_{\mu} \otimes R$ $=\mathrm{hd}_{R_{\mu}} M_{\mu}$. Because $R$ is left-flat over $R_{\mu},-\otimes_{\mu} R$ takes projective resolutions over $R_{\mu}$ to projective resolutions over $R$, and we conclude that $\mathrm{hd}_{R} M_{\mu} \otimes R \leqslant \mathrm{hd}_{R_{\mu}} M_{\mu}$. On the other hand, because $R$ is right projective over $R_{\mu}$, restriction of scalars from $R$ to $R_{\mu}$ preserves projectives, and hence projective resolutions, so $\mathrm{hd}_{R} M_{\mu} \otimes R \geqslant \mathrm{hd}_{R_{\mu}} M_{\mu} \otimes R$. But $M_{\mu} \otimes R$ has $M_{\mu}$ as a direct summand over $R_{\mu}$, so $\mathrm{hd}_{R_{\mu}} M_{\mu} \otimes R \geqslant \mathrm{hd}_{R_{\mu}} M_{\mu}$, giving the desired inequality.

Bringing in Theorem 2.2, we can deduce:

COROllary 2.5. $\mathrm{r} g \mathrm{gl} \operatorname{dim} R=\sup \left(\mathrm{r} \mathrm{gl} \operatorname{dim} R_{\mu}\right)$ if this is positive, or is $\leqslant 1$ if all $R_{\mu}$ have $\mathrm{r} \mathrm{gl} \operatorname{dim}=0$.

Proof. From the preceding Corollary, $\mathrm{r} g l \operatorname{dim} R \geqslant \sup _{\mu}\left(\mathrm{r} g l \operatorname{dim} R_{\mu}\right)$. Now let $M$ be a submodule of a free right $R$-module $F$. Then by Theorem 2.2 we can write $M=\bigoplus M_{\mu} \otimes R$. Since each $M_{\mu}$ is a sub- $R_{\mu}$-module of $F$, which is projective as an $R_{\mu}$-module, we get $\mathrm{hd}_{R_{\mu}} M_{\mu} \leqslant\left(\mathrm{rgl} \operatorname{dim} R_{\mu}\right)-1$, or $=0$ if this is negative. Hence by Corollary $2.4, \operatorname{hd}_{R} M \leqslant \sup \left(\mathrm{r} g l \operatorname{dim} R_{\mu}\right)-1$, or $=0$ if this is negative, from which the result claimed follows.

(For some further observations on this formula, see $\S 13$.)

It is easy to see that the analogs of Corollaries 2.4 and 2.5 can be proved equally easily with homological and global dimensions replaced by weak dimensions, or indeed, dimensions with respect to resolutions by any class of module preserved by the types of change-of-base operations used.

Corollary 2.4 and Theorem 2.2 immediately give,

COROLlaRY 2.6. Every projective R-module has the form $\bigoplus_{M_{\mu}} \otimes R$, where each $M_{\mu}$ is a projective $R_{\mu}$-module.

(But it is generally easy to find flat $R$-modules that are not standard.)

CoRollary 2.7. If $M=\bigoplus M_{\mu} \otimes R$ and $N=\bigoplus N_{\mu} \otimes R$ are standard $R$-modules, and for all $\mu, \operatorname{Hom}_{R_{\mu}}\left(M_{\mu}, R_{\mu}\right)=\{0\}$ (equivalently: $\operatorname{Hom}_{R}(M, R)$ $=\{0\})$, then every $R$-module homomorphism $f: M \rightarrow N$ is an induced homomorphism; thus the natural injection $\Pi_{\mu} \operatorname{Hom}_{R}\left(M_{\mu}, N_{\mu}\right) \rightarrow \operatorname{Hom}_{R}(M, N)$ is a bijection.

Proof. If $f \in \operatorname{Hom}(M, N)$, then the image of each $M_{\mu}$ under $f$ must have zero component in the $R_{\mu}$-projective part of $N$, hence must lie entirely in $N_{\mu}$.

Something we would like to know is whether every extension of a standard module by a standard module is standard!

The preceding results show that the standard modules play a large role in 
the module-theory of $R$. We now turn to Theorem 2.3 for more exact information about the category of such modules. For any ring $A$, let $\operatorname{Mod} A$ denote the category of finitely generated (right) $A$-modules, and let $S_{\oplus}(\operatorname{Mod} A)$ denote the additive semigroup of isomorphism-classes, $[M]$, of objects of $\operatorname{Mod} A$, under the operation induced by direct sums: $[M]+[N]=[M \oplus M]$. Let $S t d R \subseteq \operatorname{Mod} R$ denote the full subcatogory of finitely generated $R$-modules which can be given structures of standard modules, and $S_{\oplus}(S t d R) \subseteq S_{\oplus}(\operatorname{Mod} R)$ the corresponding subsemigroup. For each $\mu$, let $q_{\mu}: S_{\oplus}\left(\operatorname{Mod} R_{0}\right) \rightarrow$ $S_{\oplus}\left(\operatorname{Mod} R_{\mu}\right)$, and $p_{\mu}: S_{\oplus}\left(\operatorname{Mod} R_{\mu}\right) \rightarrow S_{\oplus}(S t d R)$ be the semigroup homomorphisms induced by the operations $-\otimes_{0} R_{\mu}$ and $-\otimes_{\mu} R$.

(1)

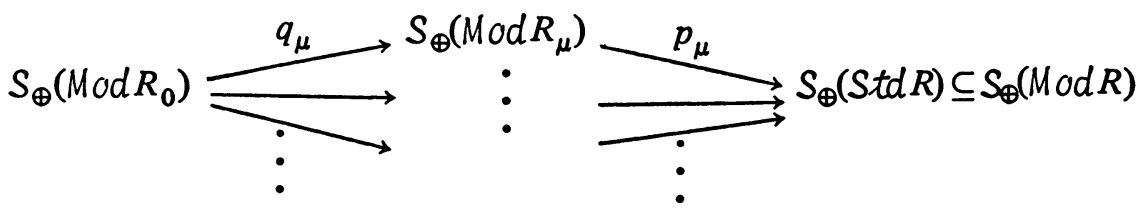

Let us first consider Theorem 2.3 in the case where $f$ is an isomorphism. It provides, in effect, a criterion for two finitely generated standard modules to be isomorphic, namely if and only if one can go from the representations of one to that of the other by a series of basic transfers. We formalize this statement (and give the proof in detail) as:

CoROllary 28. With respect to the maps $p_{\mu}$ and $q_{\mu}$, the semigroup $S_{\oplus}(S t d R)$ is the fibered sum (pushout) over $S_{\oplus}\left(\operatorname{Mod} R_{0}\right)$ of the semigroups $S_{\oplus}\left(\operatorname{Mod} R_{\mu}\right)$.

Proof. Clearly, the diagram (1) commutes.

Now the fibered coproduct of semigroups in question can be described as the quotient of the direct sum (simple coproduct), $\bigoplus_{\mu} S_{\oplus}\left(\operatorname{Mod} R_{\mu}\right)$, by the congruence relation $\sim$ generated by all relations $q_{\mu}(a) \sim q_{\mu^{\prime}}(a) \quad\left(a \in S_{\oplus}\left(\operatorname{Mod} R_{0}\right)\right.$; $\left.\mu, \mu^{\prime} \in \Lambda \cup\{0\}\right)$. Hence what we must show is that if two elements $m=$ $\Sigma\left[M_{\mu}\right]$ and $n=\Sigma\left[N_{\mu}\right]$ of $\bigoplus S_{\oplus}\left(\operatorname{Mod} R_{\mu}\right)$ have the same image in $S_{\oplus}(S t d R):$

$$
\sum p_{\mu}\left[M_{\mu}\right]=\sum p_{\mu}\left[N_{\mu}\right]
$$

in other words, if $\bigoplus M_{\mu} \otimes R \cong \bigoplus N_{\mu} \otimes R$, then these elements $m$ and $n$ are congruent under $\sim$. But indeed, given an isomorphism $f: \bigoplus M_{\mu} \otimes R \rightarrow$ $\bigoplus N_{\mu} \otimes R$, Theorem 2.3 tells us that by applying to $M$ a sequence of transvections and basic transfer isomorphisms, we can get an induced isomorphism, which corresponds to equality in $\bigoplus\left(S_{\oplus}\left(\operatorname{Mod} R_{\mu}\right)\right)$. Now a transvection does not change the element of $\bigoplus\left(S_{\oplus}\left(\operatorname{Mod} R_{\mu}\right)\right)$ associated with a standard module, 
while a basic transfer corresponds to a change of the form $p+q_{\mu}(a) \mapsto p+q_{\mu^{\prime}}(a)$. Hence $m$ and $n$ can be connected by a finite chain of such changes. I.e., $m \sim n$, as desired.

To use the general case of Theorem 2.3, let us define a preordering on each of the semigroups $S_{\oplus}\left(\operatorname{Mod} R_{\mu}\right)$ and $S_{\oplus}(S t d R)$ by writing $[N] \leqslant[M]$ if $N$ is a homorphic image of $M$. Then the theorem easily yields:

Corollary 2.9. The preordering $\leqslant$ on $S_{\oplus}(S t d R)$ is induced by the corresponding preorderings on the semigroups $S_{\oplus}\left(\operatorname{Mod} R_{\mu}\right)$. In fact, if $n=$ $\Sigma p_{\mu}\left(n_{\mu}\right) \leqslant m \in S_{\oplus}(S t d R)$, then $m$ can be written $\Sigma p_{\mu}\left(m_{\mu}\right)$ such that for all $\mu, n_{\mu} \leqslant m_{\mu}$ in $S_{\oplus}\left(\operatorname{Mod} R_{\mu}\right)$.

For certain applications, e.g., in [3], one wants to restrict one's attention to special classes of modules. Let us define an $R_{0}$-stable class of $R_{\mu}$-modules (respectively, of finitely generated $R_{\mu}$-modules) to be a possibly empty class $\mathrm{C}_{\mu}$ of $R_{\mu}$-modules, closed under isomorphisms $\left(M \cong M^{\prime} \in \mathrm{C}_{\mu} \Rightarrow M \in \mathrm{C}_{\mu}\right)$, and such that given any (finitely generated) $R_{\mu}$-module $M$ and any (finitely generated) basic $R_{\mu}$-module $P$, we have $M \in C_{\mu} \Leftrightarrow M \oplus P \in C_{\mu}$.

If we are given such a class $C_{\mu}$ for each $\mu$, let us denote by $C$ the class of $R$-modules isomorphic to (finitely generated) standard $R$-modules of the form $\bigoplus M_{\mu} \otimes R$, with each $M_{\mu} \in \mathcal{C}_{\mu}$. Proposition 2.1 and Theorem 2.2 easily give the following Corollary (except that the proof of $R_{0}$-stability of $C$ in the finitely generated case requires Theorem 2.3).

Corollary 2.10. Suppose that, for each $\mu, C_{\mu}$ is an $R_{0}$-stable class of (finitely generated) $R_{\mu}$-modules, and let $C$ be the induced class of standard $R$ modules. Then $C$ is $R_{0}$-stable, and if each $C_{\mu}$ is closed under going to (finitely generated) submodules, so is $\mathrm{C}$.

If $C_{\mu}$ is an $R_{0}$-stable class of finitely generated $R_{\mu}$-modules, let $p S_{\oplus}\left(C_{\mu}\right)$ denote the subset of $S_{\oplus}\left(\operatorname{Mod} R_{\mu}\right)$ induced by the modules of $C_{\mu}$. This will be a preordered partial semigroup. Let $\bigoplus p S_{\oplus}\left(C_{\mu}\right)$ denote the class of formal sums $\Sigma\left[M_{\mu}\right]\left(M_{\mu} \in C_{\mu}\right)$ with almost all $M_{\mu}=\{0\}$. For each $\mu$, we have an action of the abelian semigroup $S_{\oplus}\left(\operatorname{Mod} R_{0}\right)$ on $\oplus p S_{\oplus}\left(C_{\mu}\right)$, by "adding basic modules to the $\mu$ component". Let $\bigoplus^{\prime} p S_{\oplus}\left(C_{\mu}\right)$, denote the quotient of this direct sum by the least equivalence relation equalizing these actions of $S_{\oplus}\left(\operatorname{Mod} R_{0}\right)$. (If every $C_{\mu}$ contains $\{0\}$, then every $p S_{\oplus}\left(C_{\mu}\right)$ contains $q_{\mu}\left(S_{\oplus}\left(\operatorname{Mod} R_{0}\right)\right)$, and $\bigoplus^{\prime} p S_{\oplus}\left(C_{\mu}\right)$ can be described as a fibered sum (pushout) of partial semigroups over $S_{\oplus}\left(\operatorname{Mod} R_{0}\right)$. If each $C_{\mu}$ is closed under $\oplus$, then the qualifier "partial" can be deleted.) We now get from Corollaries 2.8 and 2.9: 
Corollary 2.11. Suppose that, for each $\mu, C_{\mu}$ is an $R_{0}$-stable class of finitely generated $R_{\mu}$-modules, and $C$ the induced class of standard $R$-modules. Then the natural map $\bigoplus^{\prime} p S_{\oplus}\left(C_{\mu}\right) \rightarrow p S_{\oplus}(C)$ is a bijection, and the partial operation + and preorder $\leqslant$ on $p S_{\oplus}(C)$ are precisely those induced by the corresponding structure on the $p S_{\oplus}\left(C_{\mu}\right)$.

Note that for arbitrary $\left(R_{\mu}\right)$, the projective $R_{\mu}$-modules form an $R_{0}$-stable class closed under $\oplus$. Since all projective $R$-modules are standard (Corollary 2.6), we see that the abelian semigroup of isomorphism classes of finitely generated projective $R$-modules will be the pushout of the corresponding semigroups for the $R_{\mu}$.

For another more specialized application, recall that a right fir (free, $i$ deal ring; see [16]) can be characterized as a ring $A$ such that the class of free right $A$-modules is closed under submodules, and the semigroup $S_{\oplus}$ (Free $A$ ) of finitely generated free right $A$-modules is isomorphic to the additive semigroup of nonnegative integers. Such a ring is an integral domain, hence if in our present context, some $R_{\mu}$ is a right fir, $R_{0}$ must be a sfield, and the map $S_{\oplus}\left(\operatorname{Mod} R_{0}\right) \rightarrow S_{\oplus}\left(\right.$ Free $\left.R_{\mu}\right)$ will be an isomorphism. We can easily deduce:

CoROllaRy 2.12 (CF. $[12, \S 6])$. A coproduct of right firs over a sfield is a right fir.

Our results can also be applied to non- $R_{0}$-stable classes. Suppose that, for some $n>0$, all our $R_{\mu}$ are $n$-firs (again see [16]). Then it is easy to see that the class of standard $R$-modules of the form $\bigoplus F_{\mu} \otimes R$, where each $F_{\mu}$ is free over $R_{\mu}$, and their ranks sum to $n$, is closed under free transfer isomorphisms. It is now easy to recover:

Corollary 2.13 (Cohn [14], [1, p. 202]). A coproduct of $n$-firs over a sfield is an $n$-fir.

(For some details of proof cf. next corollary.)

In [2], we generalized the idea of a free module of rank $n$ over an $n$-fir to that of a "hereditarily" projective module over an arbitrary ring $R$-a projective $R$-module $P$ such that the image of $P$ under any homomorphism into a free module of finite rank, $f: P \rightarrow F$, is again projective.( $\left.{ }^{3}\right)$ Corollary 2.13 generalizes to:

(3) Note. Azumaya's students H. S. Ahluwalia and M. S. Shrikhande use some similar terms with quite different meanings. 
Corollary 2.14. A finitely generated standard R-module $M=\bigoplus M_{\mu} \otimes R$ is hereditarily projective if and only if for every standard R-module structure $\bigoplus M_{\mu}^{\prime} \otimes R$ that can be obtained from the given one by basic transfers, each $M_{\mu}^{\prime}$ is a hereditarily projective $R_{\mu}$-module.

SKETCH OF PROOF. “ $\Rightarrow$ " If some $M_{\mu}^{\prime}$ had a nonprojective image under a map into a projective $R_{\mu}$-module, $f: M_{\mu}^{\prime} \rightarrow P$, then $M$ would have a nonprojective image under the induced map $M \rightarrow P \otimes_{\mu} R$. To prove “ $\Leftarrow$ ", note that by Theorem 2.2, the image of a map of $M$ into a free $R$-module will be a standard module $N$, then apply Theorem 2.3 to the map of $M$ into $N$, and use the facts that the $M_{\mu}^{\prime}$ will be hereditarily projective, and that $N$, and hence the $N_{\mu}$, are submodules of a projective module.

(By duality [2, Lemma 2.15], the same result thus holds for finitely generated cohereditarily projective modules. It is not hard to prove the same result for finitely generated weakly hereditarily projective modules; we do not know what may be true of strongly hereditarily projective modules $[2, \S 2]$.)

We now turn to the theory of the general linear group. It would be nice if we could say that $\mathrm{GL}_{n}$ of our coproduct $R$ was generated by its subgroups $\mathrm{GL}_{n}\left(R_{\lambda}\right)$, and elementary matrices. It will turn out instead that they are generated by the $\mathrm{GL}_{n}\left(R_{\lambda}\right)$ and certain matrices corresponding to our transvections. Only when our rings are $n$-firs can we reduce the latter to elementary matrices.

If $\gamma$ and $\epsilon$ are a column and a row vector of length $n$ over some $R_{\mu}$, with $\epsilon \gamma=0$, and $\delta$ any element of $R$, let us (for the purposes of the next corollary) call the invertible $n \times n$ matrix $\theta=I-\gamma \delta \epsilon$ a " $\mu$-based transvection". Note that a nondiagonal elementary matrix over $R$ is in particular a 0 based transvection matrix.

One of the equivalent conditions for $R_{\mu}$ to be an $n$-fir [16, Theorem 1.1] is that for any row and column vectors $\epsilon, \gamma$ over $R$ of length $n$, with $\epsilon \gamma=$ 0 , there should exist $\beta \in \mathrm{GL}_{n}\left(R_{\mu}\right)$ such that $\epsilon \beta$ and $\beta^{-1} \gamma$ have the blockforms $(0, *)$ and $\left(\begin{array}{l}* \\ 0\end{array}\right)$ with blocks of corresponding sizes. In that case, the $\mu$-based transvection $\theta=I-\gamma \delta \epsilon$ as above is conjugate to $\beta^{-1} \theta \beta=I+\left(\begin{array}{ll}0 & * \\ 0 & 0\end{array}\right)$, which is a product of elementary matrices.

Corollary 2.15. Suppose $R_{0}$ is a sfield, and $n$ an integer such that for all $\mu$, all $m \leqslant n$, and all $R_{\mu}$-modules $M_{\mu}$, we have $M_{\mu} \oplus R_{\mu}^{m} \cong R_{\mu}^{n} \Rightarrow$ $M_{\mu} \cong R_{\mu}^{n-m}$ (isomorphisms as $R_{\mu}$-modules). Then $\mathrm{GL}_{n}(R)$ is generated by its subgroups $\mathrm{GL}_{n}\left(R_{\mu}\right)$ and the $\mu$-based transvections $(\mu$ ranging over $\Lambda \cup$ $\{0\})$.

In particular (CoHN [14, last paragraph], also [1,p. 202]) if all $R_{\mu}$ are $n$-firs, $\mathrm{GL}_{n}(R)$ is generated by the $\mathrm{GL}_{n}\left(R_{\mu}\right)$ and the elementary matrices. 
SKETCH OF PROOF. We apply Theorem 2.3 to an automorphism $f$ of the free $R$-module of rank $n, M=N=R^{n}=R_{0}^{n} \otimes_{0} R$. Our module-theoretic hypotheses insure that at every step in the transformations of $M$ given by that theorem, the "components" $M_{\mu}$ will all be free and have ranks summing to $n$. Hence we can always keep an $n$-element basis of $M$ formed from bases of the current $M_{\mu}$.

When we perform a free transfer, splitting off a summand $x R_{\mu_{1}}$ from some $M_{\mu_{1}}$, and attaching $x R_{\mu_{2}}$ to $M_{\mu_{2}}$, let us precede this by changing our basis of $M_{\mu_{1}}$ to one of the form $B^{\prime} \cup\{x\}$, where $B^{\prime}$ is a basis of the submodule $M_{\mu_{1}}^{\prime}$ complementing $x R_{\mu_{1}}$ (cf. $\S 1$ ). This change of basis corresponds to the action of an element of $\mathrm{Gl}_{n}\left(R_{\mu_{1}}\right)$. The free transfer itself then becomes a formal redistribution of the basis elements among the $M_{\mu}$.

When we apply a transvection $\theta$, if the indices $\mu_{1}$ and $\mu_{2}$ involved (§1) are distinct, then the matrix representing $\theta$ will essentially have the form $\left(\begin{array}{ll}I & * \\ 0 & I\end{array}\right)$, a product of elementary matrices, which are 0 -based transvection matrices, while if $\mu_{1}=\mu_{2}$, it will be a $\mu_{1}$-based transvection matrix.

Theorem 2.3 tells us that after being composed with these automorphisms, $f$ yields an induced automorphism $f^{\prime}$. In view of the structure of standard module we chose for $N$, this means $f^{\prime} \in \mathrm{GL}_{n}\left(R_{0}\right)$. So $f$ is a composition of matrices of the sorts described.

The final assertion follows from our observations on $\mu$-based transvections when $R_{\mu}$ is an $n$-fir.

Corollary 2.16 (UNITS AND ZERO-DIVISORS). Suppose $R_{0}$ is a sfield, and that for all $\mu$ and all $R_{\mu}$-modules $M_{\mu}$ we have $M_{\mu} \oplus R_{\mu} \cong R_{\mu} \Rightarrow M_{\mu}=$ $\{0\}$ (isomorphism as $R_{\mu}$-modules. Equivalently, any one-sided invertible element of $R_{\mu}$ is invertible.) Then:

(i) The group of units of $R$ is generated by the units of the $R_{\mu}$, together with $\left\{1-\gamma \delta \epsilon \mid \epsilon \gamma=0 ; \epsilon, \gamma \in R_{\mu}, \mu \in \Lambda \cup\{0\} ; \delta \in R\right\}$.

(ii) If $x y=0$ in $R$, then there exist a unit $\alpha$ in $R$, and sets $U, V$ in some $R_{\mu_{1}}$ with $U V=\{0\}$, such that $x \in R U \alpha$, and $y \in \alpha^{-1} V R$.

Proof. (i) is simply the case $n=1$ of the preceding corollary.

To prove (ii), consider the homomorphism $f=$ left multiplication by $x$ : $M=R \rightarrow R$, which has $y$ in its kernel. Apply Theorem 2.3 to $f$ as a map of $M$ onto the standard module $N=f(R)$. By our module-theoretic hypotheses (cf. proof of preceding result), the standard module $M^{\prime} \cong M$ we get will have the form $R_{\mu_{1}} \otimes R$ for some $\mu_{1}$ (all other components zero). Adjusting by the isomorphism $M \cong M^{\prime}$, which in terms of the natural bases for $M$ and $M^{\prime}$ takes the form of multiplication by a unit $\alpha \in R$-in other words, replacing $x$ 
by $x \alpha$ and $y$ by $\alpha^{-1} y$-we are reduced to the case where $f: M \rightarrow N$ is an induced homomorphism, and the standard structure on $M$ is $M=R_{\mu_{1}} \otimes R$.

Now the significance for us of the fact that $f$ is an induced homomorphism will be that $\operatorname{Ker} f$ is generated by the kernels of the maps $f_{\mu}: M_{\mu} \rightarrow N_{\mu}$-in this case, by the kernel of the one map $f_{\mu_{1}}: R_{\mu_{1}} \rightarrow N_{\mu_{1}} \subseteq N \subseteq R$. Call this kernel (a right ideal of $R_{\mu_{1}}$ ) V, and its left annihilator in $R_{\mu_{1}}, U$. Thus, $y \in \operatorname{Ker} f=\left(\operatorname{Ker} f_{\mu_{1}}\right) R=V R$, while by the fact that $R$ is right flat over $R_{\mu_{1}}$, and $0=f_{\mu_{1}}(V)=x V$, in $R$, we must have $x \in R U$.

Incidentally, it is not hard to verify by Corollary 2.8 that the moduletheoretic conditions in the hypotheses of the above two corollaries will carry over to the coproduct ring $R$.

The technique used in the above proof, of first observing by Theorem 2.2 that the image of a map $f$ of standard modules is standard, then applying Theorem 2.3 to the surjection $f: M \rightarrow f(M)$ to get a description of the kernel, deserves to be abstracted as a final corollary:

CoRollary 2.17. Let $f: M \rightarrow N$ be a homomorphism of standard $R$ modules. Then there exists an isomorphism $\alpha: M^{\prime} \cong M$ of standard modules (namely the one given in Theorem 2.3) such that putting $f \alpha=f^{\prime}: M^{\prime} \rightarrow N$, we have $\operatorname{Ker} f^{\prime}=\bigoplus\left(\operatorname{Ker} f^{\prime} \mid M_{\mu}^{\prime}\right) \otimes R$.

3. Preview of the proofs. In $\S \S 4-8$ below, $R_{0}$ will be assumed a sfield.

In $\S 4$, we obtain a normal form for elements of a standard $R$-module $N=$ $\bigoplus N_{\mu} \otimes R$ (which will immediately give us Proposition 2.1).

We then consider an arbitrary family $\left(L_{\mu}\right)$ where, for each $\mu, L_{\mu}$ is an $R_{\mu}$-submodule of $N$. We should like the submodule of $N$ generated by this family to be isomorphic to $\bigoplus L_{\mu} \otimes R$. This will, in fact, be true if the elements of the $L_{\mu}$, expressed in our normal form, are such that no bad "interaction" can occur among them under the $R$-module structure of $N$. We shall write down such noninteraction conditions explicitly, call $\left(L_{\mu}\right)$ "well-positioned" (Definition 5.2) if they hold, and prove the submodule of $N$ generated by a well-positioned family $\left(L_{\mu}\right)$ is indeed isomorphic to $\bigoplus L_{\mu} \otimes R$ (Proposition 8.2). We find further (Proposition 8.4) that if a well-positioned family $\left(L_{\mu}\right)$ generates all of $N$, then we must have precisely $N_{\mu}=L_{\mu}$ for all $\mu$.

What if a family $\left(L_{\mu}\right)$ is not well-positioned? We shall show in $\S 6$ that it can then be modified to a slightly "better" family $\left(L_{\mu}^{\prime}\right)$ generating the same submodule, and that if $\left(L_{\mu}\right)$ is "finitely generated" (all $L_{\mu}$ finitely generated, and all but finitely many equal to zero), then a finite sequence of such adjustments will yield a well-positioned family. Suppose further that each $L_{\mu}$ is given as the image of some homomorphism of $R_{\mu}$-modules, $f_{\mu}: M_{\mu} \rightarrow N$, 
so that the $R$-submodule generated is the image of a map $f: M=\bigoplus M_{\mu} \otimes R \rightarrow$ $N$. Then the "adjustments" of $\left(L_{\mu}\right)$ described above correspond to transvections and free transfer automorphisms of $M$. These results (Proposition 6.2) combined with Proposition 8.4 described above, clearly yield Theorem 2.3. We also prove (Proposition 7.1) that every submodule of $N$, not necessarily finitely generated, has a well-positioned generating family of submodules. Combined with Proposition 8.2, this yields Theorem 2.2. (Combining Propositions 6.2 and 8.2 yields a direct proof of Corollary 2.17.)

The methods used here were inspired by those introduced by P. M. Cohn to show that a ring with $n$-term weak algorithm is an $n$-fir [15], [16], and that a ring with full weak algorithm is a fir [11], [12], [16]. In the first case, one repeatedly modifies an $m$-tuple $(m \leqslant n)$ of generators of a right ideal $I$ till one gets an $m$-tuple whose nonzero entries are $v$-independent and hence linearly independent. In the second case one shows that one can choose a $v$-independent generating set for an arbitrary right ideal $I$. Our condition of being "well-positioned" occupies the place of Cohn's " $v$-independence". Cohn's method, as he observes in [16], goes back to Euclid's algorithm [17, Book VII, Propositions 1 and 2] and its generalization to polynomials by Simon Stevin [23, Book II, Problem LIII].

4. Normal form in a standard module. We now take $R_{0}$ to be a skew field. Hence each $R_{\lambda}$ is free as a right $R_{0}$-module, and we can choose for it a right $R_{0}$-basis of the form $T_{\lambda} \cup\{1\}\left(1 \notin T_{\lambda}\right)$.

For each $\mu \in \Lambda \cup\{0\}$, let $N_{\mu}$ be a right $R_{\mu}$-module, and let us likewise choose for each of these modules a right $R_{0}$-basis $S_{\mu}$. We shall write $T$ for the disjoint union of the $T_{\lambda}$, and $S$ for the disjoint union of the $S_{\mu}$, and shall say that an element of $T$ or $S$ is "associated" with an index $\lambda \in \Lambda$ if it comes from the corresponding $T_{\lambda}$ or $S_{\lambda}$. (Note that elements of $S_{0}$ are not considered to be associated with any index. The idea is that the elements associated with a given index are those that will "interact", and so must be paid special attention to, when we multiply by an element of the corresponding ring. But since $S_{0}$ is a basis of $N_{0}$ over $R_{0}$, we have no "interaction" to worry about in that case.)

We claim that the standard $R$-module $\bigoplus N_{\mu} \otimes R$ has for a right $R_{0}$-basis the set of all products $s t_{1} \cdots t_{n}\left(s \in S, t_{i} \in T, n \geqslant 0\right)$ such that no two successive terms among $s, t_{1}, \cdots, t_{n}$ are associated with the same index $\lambda$.

To show this, let $U$ denote the set of all such formal products, and let $N$ denote the free right $R_{0}$-module on the set $U$. We shall give $N$ a structure of right $R$-module, and show that this has the universal property characterizing $\oplus_{N_{\mu}} \otimes R$ 
To make an $R_{0}$-module $N$ into an $R$-module, it suffices to define on it, for each $\lambda$, a right $R_{\lambda}$-module structure extending the $R_{0}$-module structure. (This follows from the definition of a coproduct of $R_{0}$-rings, and the characterization of a module over a ring $R$ as an additive group $N$ with a homomorphism of $R$ into End $(N)$.)

For any $\mu \in \Lambda \cup\{0\}$, let us call an element of $U$ "associated with $\mu$ " if and only if its last factor (an element of $S$ or $T$ ) is associated with $\mu$, and let us denote by $U_{\sim \mu}$ the set of elements of $U$ not associated with $\mu$. Note that $U_{\sim 0}=U$.

For each index $\lambda \in \Lambda$, let us write $N$ as the direct sum of the free right $R_{0}$-module spanned by $S_{\lambda}$, and the free right $R_{0}$-module spanned by all other elements of $U$. The first submodule may be identified with $N_{\lambda}$, and thus given $N_{\lambda}$ 's structure of $R_{\lambda}$-module. Now each of the remaining elements of $U$ can be written uniquely $u \tau$, where $u \in U_{\sim \lambda}$, and $\tau$ is either the "empty word", or a member of $T_{\lambda}$. But since $\{1\} \cup T_{\lambda}$ is a basis for $R_{\lambda}$, we can identify the $R_{0}$-module spanned by all these elements with the free right $R_{\lambda}$-module on the basis $U_{\sim \lambda}$. Together, these definitions give us a structure of right $R_{\lambda}$-module on the $R_{0}$-module $N$. Since we have this for each $\lambda$, we have a structure of right $R$-module.

To show that $N$ has the universal property of the standard module $\bigoplus_{N_{\mu}} \otimes R(\S 1)$, let $f_{\mu}: N_{\mu} \rightarrow P(\mu \in \Lambda \cup\{0\})$ be any family of $R_{\mu^{-}}$ module homomorphisms of the $N_{\mu}$ into an $R$-module $P$. We define $f: N \rightarrow$ $P$ first on $S$, by $f(s)=f_{\mu}(s)\left(s \in S_{\mu}\right)$, then on $U$, by $f\left(s t_{1} \cdots t_{n}\right)=$ $f(s) t_{1} \cdots t_{n}$, and finally on $N$ by $R_{0}$-linearity. It is easy to show that this map is $R_{\lambda}$-linear for all $\lambda$, and so is $R$-linear. So we have:

Proposition 4.1 (CF. [9], [22], [4, §7]). The standard R-module $N=$ $\bigoplus N_{\lambda} \otimes R$ has for a right $R_{0}$-basis the set $U$ of products $s_{1} \cdots t_{n}$ with $s \in S, t_{i} \in T, n \geqslant 0$, and no two successive factors associated with the same index $\lambda \in \Lambda$.

For each $\lambda \in \Lambda, N$ is the direct sum as a right $R_{\lambda}$-module of $N_{\lambda}$ (which is embedded in $N$ under the canonical map), and a free $R_{\lambda}$-module with basis $U_{\sim \lambda}$.

In particular, we have proved Proposition 2.1 (for $R_{0}$ a sfield). We shall call the elements of the basis $U$ of $N$ monomials.

Given $\mu \in \Lambda \cup\{0\}$ and $u \in U_{\sim \mu}$, we shall denote by $c_{\mu u}: N \rightarrow R_{\mu}$ the $R_{\mu}$-linear "right coefficient of $u$ " function, in terms of the representation of $N$ given in the first paragraph of Proposition 4.1 for $\mu=0$, and the second paragraph for $\mu \neq 0$. 
There is a slight modification of Proposition 4.1 that we shall need at a later point:

Lemma 4.2. Let $M_{0}$ be a right $R_{0}$-module with basis $Q_{0}$, and suppose also that for each $\lambda \in \Lambda$ we are given another $R_{0}$-basis of $M_{0}, Q_{0, \lambda}$. Then a right $R_{0}$-basis for $M_{0} \otimes R$ is given by the set of all products $q t_{1} \cdots t_{n}$, where $t_{i} \in T, n \geqslant 0$, no two successive $t_{i}$ are associated with the same index, and if $t_{1}$ is associated with $\lambda \in \Lambda$, then $q \in Q_{0, \lambda}$, while if $n=0, q \in Q_{0}$.

The proof is essentially as for Proposition 4.1, except that in defining the $R_{\lambda}$-module structures on the $R_{0}$-module $M$ spanned by these products, one considers, for each $\lambda$, a different basis of the submodule $M_{0} \subseteq M$.

(Note. The arguments of the above section do not really require that $R_{0}$ be a sfield, but merely that the $R_{\lambda} / R_{0}$, the $N_{\mu}$, and $M_{0}$ all be free as right $R_{0}$-modules.)

5. Support, degree, well-ordering, purity, leading terms, and well-positioned families of submodules. Let $N$ be as in the preceding section.

For $\mu \in \Lambda \cup\{0\}$, we shall define the $\mu$-support of an element or subset of $N$ (relative to the basis we have constructed) to be the set of elements of $U_{\sim \mu}$ occurring in the $R_{\mu}$-free part of the representation of these elements described in Proposition 4.1. In other words, $u \in U_{\sim \mu}$ lies in the $\mu$-support of an element $x \in N$ if and only if $c_{\mu u}(x) \neq 0$. For $\mu \neq 0, x$ has empty $\mu$ support if and only if $x \in N_{\mu}$; for $\mu=0$, if and only if $x=0$. The 0-support of an element will also simply be called its support. Note that any finitely generated $R_{\mu}$-submodule of $N$ has finite $\mu$-support.

We define the degree of a monomial (an element of $U$ ) to mean its length, and the degree of a nonzero element of $N$ as the maximum of the degrees of the elements of its support.

Let us well-order the sets $S$ and $T$ arbitrarily. A lexicographic wellordering (reading from the left) is induced on the set of momomials of each degree. If we further consider a monomial of greater degree to be greater than any monomial of lower degree, we get a well-ordering on the whole of $U$.

By the leading term of a nonzero element of $N$, we shall mean the maximal monomial in its support, under this ordering.

Let us call a nonzero element of $N \lambda$-pure $(\lambda \in \Lambda)$ if all of its terms of maximal degree are associated with the index $\lambda$. If $x$ is an element of $N$ that is not $\lambda$-pure, some of the monomials of maximal degree in the support of $x$ will lie in $U_{\sim \lambda}$; let us call the greatest of these the $\lambda$-leading term of $x$. (Thus, $\lambda$-leading term is only defined for non- $\lambda$-pure elements!) 
LEMmA 5.1. Let $y \in N, t_{1} \in T_{\lambda_{1}}, \cdots, t_{n} \in T_{\lambda_{n}}$, where $n>0, y$ is non- $\lambda_{1}$-pure, and successive $\lambda_{i}$ are distinct. Let $y$ be of degree $m$, with $\lambda_{1}$ leading term $u$, and suppose $u$ occurs in $y$ with coefficient $1 \in R_{0}$. Then $y t_{1} \cdots t_{n}$ is $\lambda_{n}$-pure, and has leading term $u t_{1} \cdots t_{n}$, again with coefficient 1 .

Proof. By induction, it suffices to prove the result when $n=1$. We shall write $t$ for $t_{1}, \lambda$ for $\lambda_{1}$.

Let $\dot{v}$ be any monomial other than $u$ occurring in $y$, say with coefficient $c \in R_{0}$. Note that $(v c) t \in v R_{\lambda}$. If $v$ (unlike $u$ ) is associated with the index $\lambda$, we see that $v c t \in v R_{\lambda}$ will have degree $<m+1=$ degree $u t$. If $v$ is associated with another index, then $v<u$ because $u$ is the $\lambda$-leading term of $y$. The product $v c t \in v R_{\lambda}$ will be an $R_{0}$-linear combination of terms $v \tau, \tau \in T_{\lambda} \cup\{1\}$. These will be $<u t$ by the nature of our ordering on $U$. Hence $u t$, which occurs with coefficient 1 , will be the leading term of $y t$. Further, those terms $v \tau$ which have degree $m+1$ (i.e., $\tau \neq 1$ ) are, like $u t$, associated with the index $\lambda$. So $y t$ is $\lambda$-pure.

We shall call an element of $N 0$-pure if it is not $\lambda$-pure for any $\lambda \in \Lambda$, or if it is 0 ; and the 0-leading term of a non-0-pure element of $N$ will mean its leading term. Note that a $\lambda$-pure element of degree $n$ has $\lambda$-support consisting of monomials of degrees $<n$, while a 0 -pure element's 0 -support consists of monomials of degrees $\leqslant n$.

We can now define a "well-positioned" family of submodules. It is a rather technical set of conditions, to which I was propelled by the nature of the proof; I hope that the arguments of the next few sections will give the reader some sense of why they are the "right" conditions to aim for.

DEFINITION 5.2. Suppose that for each $\mu \in \Lambda \cup\{0\}$ we are given an $R_{\mu}$-submodule $L_{\mu} \subseteq N$. We shall say that the family of submodules $\left(L_{\mu}\right)$ is well-positioned if for each $\mu$, it satisfies:

$\left(\mathrm{a}_{\mu}\right)$ All members of $L_{\mu}$ are $\mu$-pure, and for each $\mu_{1}, \mu_{2}$;

$\left(\mathrm{b}_{\mu_{1}, \mu_{2}}\right)$ The $\mu_{1}$-support of $L_{\mu_{1}}$ contains no monomial $u$ which is also the $\mu_{1}$-leading term of a non- $\mu_{1}$-pure element $x a$, where $x \in L_{\mu_{2}}, a \in R$, and where if $\mu_{1}=\mu_{2}$, degree $x a>$ degree $x$ as well.

6. Adjusting a homomorphism $f$. Suppose we are given a homomorphism $f: M \rightarrow N$ of standard $R$-modules, such that the system of $R_{\mu}$-submodules $f\left(M_{\mu}\right) \subseteq N$ is not well-positioned. We shall now describe how to modify $f$ and $M$ so as to apparently improve things somewhat. We then make this idea of improvement rigorous, for $M$ finitely generated, by associating to every 
finitely generated family $\left(L_{\mu}\right)$ of $R_{\mu}$-submodules of $N$ an index in a certain well-ordered set, and showing that the "adjustments" described lower this index. By induction, some finite sequence of such adjustments will reduce $f$ to a map $f^{\prime}: M^{\prime} \rightarrow N$ such that $\left(f^{\prime}\left(M_{\mu}^{\prime}\right)\right)$ is well-positioned.

Suppose first that $\left(f\left(M_{\mu}\right)\right)$ does not satisfy condition $\left(\mathrm{a}_{\lambda}\right)$ for some $\lambda \in$ $\Lambda$. Then $f\left(M_{\lambda}\right)$ contains an element $f(x)$, say of degree $n$, which is not $\lambda$ pure: $f(x)$ involves some monomial $u \in U_{\sim \lambda}$ of degree $n$. Thus, $c_{\lambda u} f(x)$ is a nonzero element of $R_{0}$, hence invertible. Hence the $R_{\lambda}$-linear map $c_{\lambda u} f$ : $M_{\lambda} \rightarrow R_{\lambda}$ splits, and we get $M_{\lambda}=M_{\lambda}^{\prime} \oplus x R_{\lambda}$, where $M_{\lambda}^{\prime}=\operatorname{Ker}\left(c_{\lambda u} f \mid M_{\lambda}\right)$. (Here, in constrast to the preceding section, we are really using the assumption that $R_{0}$ is a sfield.)

We now transfer the free summand $x R_{\lambda}$ of $M_{\lambda}$ to $M_{0}$. Precisely, we define a free transfer isomorphism $\iota: M^{\prime} \rightarrow M$, where $M_{\lambda}^{\prime}$ is defined as above, $M_{0}^{\prime}=M_{0} \oplus x R_{0}$, and $M_{\mu}^{\prime}=M_{\mu}$ for all $\mu \neq \lambda, 0$. We then set $f^{\prime}=f i: M^{\prime}$ $\rightarrow N$. Note that the $\lambda$-support of $f^{\prime}\left(M_{\lambda}^{\prime}\right)$ no longer contains $u$ !

On the other hand, suppose $f$ fails to satisfy $\left(\mathrm{a}_{0}\right)$, so that $f\left(M_{0}\right)$ contains an element $f(x)$ (say of degree $n$ ) which is not 0-pure. This means $f(x)$ is nonzero and is $\lambda$-pure for some $\lambda \in \Lambda$. Let $u$ be any monomial of degree $n$ in the support of $f(x)$. Again, we see that $c_{0 u} f: M_{0} \rightarrow R_{0}$ splits: $M_{0}=\operatorname{Ker}\left(c_{0 u} f \mid M_{0}\right) \oplus x R_{0}$. This time, we transfer the free summand we have obtained from $M_{0}$ to $M_{\lambda}$.

If $\left(\mathrm{b}_{\mu_{1}, \mu_{2}}\right)$ fails, let $u, a$, and $f(x)$ (for " $x$ ") be as in the statement of that condition. Adjusting $a$ by a member of $R_{0}$ if necessary, we can assume $c_{\mu_{1} u}(f(x) a)=1$. Put $e=c_{\mu_{1} u} f: M_{\mu_{1}} \rightarrow R_{\mu_{1}}$, and let $\epsilon: M \rightarrow R$ be the $R$-linear map (killing all other $M_{\mu}$ ) induced by $e$. Let $\delta: R \rightarrow R$ be left multiplication by $a$; and let $\gamma: R \rightarrow M$ be the $R$-linear map taking 1 to $x$. We claim $\iota=\mathrm{id}_{M}-\gamma \delta \epsilon$ is a transvection. We have to check that, if $\mu_{1}=\mu_{2}, e(x)=0$. But in this case, $\operatorname{deg} u=\operatorname{deg} f(x) a>\operatorname{deg} f(x)$, by the statement of $\left(b_{\mu_{1}, \mu_{2}}\right)$, so $u$ cannot be in the $\mu_{1}$-support of $f(x)$, so $e(x)=$ $c_{\mu_{1} u} f(x)=0$.

Note that this $\iota$ will leave elements of $M_{\mu}$ fixed for all $\mu \neq \mu_{1}$, while each $y \in M_{\mu_{1}}$ will be sent to the unique element of the form $y-x a c$ $\left(c \in R_{\mu_{1}}\right)$ whose $\mu_{1}$-support does not contain $u$. In general, this $\mu_{1}$-support will contain some elements $v$ that were not in the $\mu_{1}$-support of $y$, but note that because $u$ is the $\mu_{1}$-leading term of $x a$, all such $v$ are $<u$ under our ordering.

We now want to show that using the above operations, we eventually arrive somewhere.

Let us take two disjoint copies of $U$, which we shall call $U_{0}$ and $U_{\Lambda}$, and whose elements will be denoted, respectively, $u_{0}$ and $u_{\Lambda}(u \in U)$. We 
order the union $U_{0} \cup U_{\Lambda}$ by "interlacing" these copies, so that:

(el'ts of $U_{0}$ of $\left.\operatorname{deg} 1\right)<\left(\right.$ el'ts of $U_{\Lambda}$ of $\operatorname{deg} 1$ )

$$
\left.<\cdots<\text { (el'ts of } U_{0} \text { of } \operatorname{deg} n\right)<\left(\text { el'ts of } U_{\Lambda} \text { of } \operatorname{deg} n\right)<\cdots \text {. }
$$

Here $U_{0}$ and $U_{\Lambda}$ each keep the internal ordering of $U$. Clearly, $U_{0} \cup U_{\Lambda}$ is well-ordered.

Let $H$ denote the additive semigroup of almost everywhere zero, nonnegative integer-valued functions on $U_{0} \cup U_{\Lambda}$, ordered lexicographically reading from higher to lower members of $U_{0} \cup U_{\Lambda}$. Then $H$ is well-ordered, because $U_{0} \cup U_{\Lambda}$ is. (Cf. [13, Theorem III.2.9].)

If $\left(L_{\mu}\right)$ is a family of finitely generated $\left(R_{\mu}\right)$-modules, only finitely many of which are nonzero, let the index $h\left(\left(L_{\mu}\right)\right) \in H$ of this system be defined as follows: For each $u \in U$, the value of $h\left(\left(L_{\mu}\right)\right)$ at $u_{0}$ will be 1 if $u$ lies in the 0-support of $L_{0}, 0$ otherwise. Its value at $u_{\Lambda}$, on the other hand, will equal the number of $\lambda \in \Lambda$ for which $u$ is in the $\lambda$-support of $L_{\lambda}$. This will indeed be almost everywhere zero, because each $L_{\mu}$ has finite $\mu$-support.

We now observe that the free transfer isomorphism $\iota$ we performed when condition $\left(\mathrm{a}_{\lambda}\right)$ failed had the effect of cutting down the module $M_{\lambda}$, and in particular, eliminating $u$ from the $\lambda$-support of $f\left(M_{\lambda}\right)$, thus decreasing $h\left(\left(f\left(M_{\lambda}\right)\right)\right)$ at $u_{\Lambda}$. It may also increase the index at certain points of $U_{0}$, since $M_{0}$ was enlarged; but since these are all of degree $\leqslant n$, they will be below $u_{\Lambda}$ in $U_{0} \cup U_{\Lambda}$. So by our choice of ordering of $H$, the new index $h\left(\left(f^{\prime}\left(M_{\mu}^{\prime}\right)\right)\right)$ is less than the old value $h\left(\left(f\left(M_{\mu}\right)\right)\right)$.

The transfer we performed when $\left(\mathrm{a}_{0}\right)$ ) failed decreased our index at $u_{0}$, while the values at which it may have increased it are all elements of $U_{\Lambda}$ of degree $<n$, because a $\lambda$-pure element of degree $n$ has $\lambda$-support consisting of elements of degrees $\leqslant n-1$. So again our index was lowered.

Finally, in the $\left(b_{\mu_{1}, \mu_{2}}\right)$ case, it is clear from the observations we made on the support of $f(y) \quad\left(y \in M_{\mu_{1}}\right)$ that our index has been decreased at a certain point $\left(u_{\Lambda}\right.$ or $u_{0}$ depending on $\mu_{1}$ ), and possibly increased only at smaller values. (Incidentally, this is the one case in which we used the full ordering on $U$. In the other cases, comparison by length was all that was involved.) We have proved:

LEMma 6.1. If $f: M \rightarrow N$ is a homomorphism of standard $R$-modules such that $M$ is finitely generated, and $\left(f\left(M_{\mu}\right)\right)$ is not well-positioned, then there exists either a free transfer isomorphism $\iota: M^{\prime} \rightarrow M$, or a transvection automorphism เ of $M=M^{\prime}$, such that $h\left(\left(f\left(M_{\mu}^{\prime}\right)\right)\right)<h\left(\left(f\left(M_{\mu}\right)\right)\right)$.

Hence by induction on $h\left(\left(f\left(M_{\mu}\right)\right)\right)$, we get: 
Proposition 6.2. If $f: M \rightarrow N$ is a homomorphism of standard R-modules, and $M$ is finitely generated, then there exists an $\alpha: M^{\prime} \stackrel{\sim}{\rightarrow}$, which is a finite composition of free transfer isomorphisms and transvection automorphisms, such that the system $\left(f \alpha\left(M_{\mu}^{\prime}\right)\right)$ of submodules of $N$ is well-positioned.

\section{Generating families for arbitrary submodules $L$.}

Proposition 7.1. Let $L$ be an $R$-submodule of the standard $R$-module $N$. Then there exists a well-positioned family of $R_{\mu}$-submodules $L_{\mu} \subseteq L \subseteq N$, such that $L=\Sigma L_{\mu} R$.

Proof. For each $\mu$, let $X_{\mu}$ denote the set of elements of $U_{\sim \mu}$ which appear as $\mu$-leading terms of non- $\mu$-pure members of $L$. Then we define each $L_{\mu}$ to be the $R_{\mu}$-submodule of $L$ consisting of all elements whose $\mu$-support is disjoint from $X_{\mu}$.

It is immediate from Definition 5.2 that $\left(L_{\mu}\right)$ is well-positioned!

We want to show that the $L_{\mu}$ generate $L$. For any $y \in N$, and any $\mu_{1}$, let $h\left(y, \mu_{1}\right) \in H$ denote the characteristic function of the $\mu_{1}$-support of $y$, taken in $U_{0}$ if $\mu_{1}=0$, in $U_{\Lambda}$ if $\mu_{1} \in \Lambda$. (One may think of $h\left(y, \mu_{1}\right)$ as the index of the system of submodules of $N$ defined to have $\mu_{1}$-component $y R_{\mu_{1}}$, and other components zero.)

If the $L_{\mu}$ do not generate $L$, let $y$ be a member of $L$ not in $\Sigma L_{\mu} R$, and $\mu_{1}$ a member of $\Lambda \cup\{0\}$, chosen together so as to minimize $h\left(y, \mu_{1}\right)$. From this minimality assumption applied to the choice of $\mu_{1}$, one sees that $y$ must be $\mu_{1}$-pure. Since $y \notin L_{\mu_{1}}$, the $\mu_{1}$-support of $y$ contains some monomial $u \in X_{\mu_{1}}$, that is, the $\mu_{1}$-leading term $u$ of some non- $\mu_{1}$-pure element $x \in L$. We may take $u$ to occur with coefficient 1 in $x$.

Note that if $\mu_{1}=0$, then non- $\mu_{1}$-purity means that $x$ is $\lambda$-pure for some $\lambda$, hence $h(x, \lambda)<h(y, 0)$, since the $\lambda$-support of $x$ will consist of elements of degrees $<$ degree $x=$ degree $u \leqslant$ degree $y$. If on the other hand $\mu_{1} \in \Lambda$, then one similarly sees by our ordering of $H$ that $h(x, 0)<h\left(y, \mu_{1}\right)$. In either case, our minimality assumption tells us that $x \in \Sigma L_{\mu} R$.

But now writing $c=c_{\mu_{1} u}(y) \in R_{\mu_{1}}$, we have $h\left(y-x c, \mu_{1}\right)<h\left(y, \mu_{1}\right)$, so $y-x c \in \Sigma L_{\mu} R$, It follows that $y \in \Sigma L_{\mu} R$, contradicting our assumption.

8. The submodule generated by a well-positioned system. In this section we shall discover the virtues of well-positioned systems.

Let $\left(L_{\mu}\right)$ be a well-positioned system of $R_{\mu}$-submodules of $N$.

Given $\mu \in \Lambda \cup\{0\}$, let us choose for each monomial $u$ that is the leading term of some element of $L_{\mu}$ an element $q \in L_{\mu}$ having this leading 
term, with coefficient 1 . For each $\mu$, call the set of $q$ 's so selected $Q_{\mu}$. It follows from the well-ordering of $U$ that $Q_{\mu}$ is an $R_{0}$-basis of $L_{\mu}$.

The leading term of each $q \in Q_{\mu}$ will also be called its "key term". Note that if $\mu \neq 0$, this term is also the $\mu^{\prime}$-leading term of $q$ for all $\mu^{\prime} \neq \mu$. An element of $L_{0}$, being 0 -pure, likewise has a $\lambda$-leading term for all $\lambda \in \Lambda$, but for at least one $\lambda$, this will not equal its leading term; so we must do a little more work in this case: Given $\lambda \in \Lambda$, we choose for each monomial $u$ that is the $\lambda$-leading term of a member of $L_{0}$, an element $q \in L_{0}$ having $u$ as $\lambda$-leading term, with coefficient 1 , and call the set of $q$ so chosen $Q_{0, \lambda}$. Each of these sets will, like $Q_{0}$, be an $R_{0}$-basis of $L_{0}$. By the "key term" of a member of $Q_{0, \lambda}$, we shall mean its $\lambda$-leading term.

Now let $V$ denote the family of all products:

$$
\begin{aligned}
& q t_{1} \cdots t_{n} \quad\left(n \geqslant 0, t_{i} \in T_{\lambda_{i}}, \lambda_{i} \neq \lambda_{i+1}\right) \text {, where either: } \\
& q \in Q_{0} \quad \text { and } n=0, \\
& \text { or } q \in Q_{0, \lambda_{1}} \quad \text { and } n \geqslant 1, \\
& \text { or } q \in Q_{\lambda_{0}} \quad \text { for some } \lambda_{0} \in \Lambda \text {; and } \lambda_{0} \neq \lambda_{1} \text { if } n \geqslant 1 .
\end{aligned}
$$

Lemma 8.1. If $q t_{1} \cdots t_{n}$ is as in (2), and the key term of $q$ is $u$, then the leading term of $q t_{1} \cdots t_{n}$ is $u t_{1} \cdots t_{n}$. Further, no two such products have the same leading terms, hence the family $V$ is linearly independent over $R_{0}$.

Proof. The first assertion follows from Lemma 5.1.

To prove the second, suppose we have two distinct elements of $V$ with the same leading term. By the first assertion, these must have the forms

$$
q t_{1} \cdots t_{n}, \quad q^{\prime} t_{1}^{\prime} \cdots t_{m}^{\prime} t_{1} \cdots t_{n}
$$

with leading terms

$$
u t_{1} \cdots t_{n}=u^{\prime} t_{1}^{\prime} \cdots t_{m}^{\prime} t_{1} \cdots t_{n}
$$

Thus, $u=u^{\prime} t_{1}^{\prime} \cdots t_{m}^{\prime}$.

Case 1. $m>0$. Then the key term $u$ of $q$ equals the leading term of $q^{\prime} t_{1}^{\prime} \cdots t_{m}^{\prime}$. But $q$ and $q^{\prime}$ are taken from two of the submodules $L_{\mu_{1}}, L_{\mu_{2}}$, and this contradicts condition $\left(\mathrm{b}_{\mu_{1}, \mu_{2}}\right)$ with $x=q^{\prime}, a=t_{1}^{\prime} \cdots t_{m-1}^{\prime}$.

Case 2. $m=0$ (so $u=u^{\prime}$ ), $n>0$. Then $q$ and $q^{\prime}$ must each belong either to $Q_{0, \lambda_{1}}$, or to $Q_{\lambda}$, where $u$ is associated to the index $\lambda$. (See (2).) As they have the same key term $u=u^{\prime}$, they cannot belong to the same set by construction. But if one belongs to $Q_{0, \lambda_{1}}$ and the other to $Q_{\lambda}$, we get an immediate contradiction to the condition $\left(b_{\lambda, 0}\right)$. 
Case 3. $m=n=0$. Like Case 2, but with $Q_{0}$ for $Q_{0, \lambda_{1}}$, and 0 for $\lambda_{1}$. The asserted linear independence of $V$ follows immediately.

But from Lemma 4.2 and Proposition 4.1, we see that the set of formal products as in (2) is an $R_{0}$-basis of $\bigoplus L_{\mu} \otimes R$. Thus we get:

Proposition 8.2. Let $\left(L_{\mu}\right)$ be a well-positioned system of $R_{\mu}$-submodules of the standard $R$-module $N$. Then the natural map $\oplus L_{\mu} \otimes R \rightarrow$ $\Sigma L_{\mu} R(\subseteq N)$ is an isomorphism.

Propositions 7.1 and 8.2 immediately give Theorem 2.2: Every $R$-submodule of a standard $R$-module is isomorphic to a standard $R$-module.

Now suppose $x \in \Sigma L_{\mu} R$ has degree 1 as a member of $N$. Writing $x$ as an $R_{0}$-linear combination of elements of $V$, we see that each of these must also be of degree 1 (lie in $\Sigma N_{\mu}$ ), and hence be of length 1 as members of $V$ (lie in some $L_{\mu}$ ). If further $x$ lies in some single $N_{\mu_{1}}$, we can see that it can involve no terms from any $L_{\mu_{2}}\left(\mu_{2} \neq \mu_{1}\right)$; if it did, the $\mu_{1}$-leading term of the $L_{\mu_{2}}$-part of $x$ could not be cancelled by any of the other terms occurring. Consequently we have:

Lemma 8.3. Let $N,\left(L_{\mu}\right)$ be as above, $L=\Sigma L_{\mu} R$. Then for all $\mu_{1} \in$ $\Lambda \cup\{0\}, L \cap N_{\mu_{1}} \subseteq L_{\mu_{1}}$.

If $L=N$ this says $N_{\mu_{1}} \subseteq L_{\mu_{1}}$ for all $\mu_{1}$. But clearly if any of these inclusions were proper, this would give us a proper inclusion of standard modules, $N \subset L$ ! Hence, rather:

PROPOSITION 8.4. Let $\left(L_{\mu}\right)$ be a well-positioned system of $R_{\mu}$-submodules of the standard $R$-module $N$, such that $\Sigma L_{\mu} R=N$. Then for all $\mu$, $L_{\mu}=N_{\mu}$.

Propositions 6.2 and 8.4 give Theorem 2.3. (Take $f$ surjective, and apply Proposition 8.4 to the system $\left(f \alpha\left(M_{\mu}^{\prime}\right)\right)$ given by Proposition 6.2.)

This completes the proof of our main results, when $R_{0}$ is a sfield.

9. Finite direct products of sfields. Let $K^{(1)}, \cdots, K^{(r)}$ be rings (arbitrary for the moment), let $R_{0}=K^{(1)} \times \cdots \times K^{(r)}$, and for $j=1, \cdots, r$, let $e^{(j)}$ denote the element of $R_{0}$ with 1 in the $i$ th place and 0 's elsewhere. Thus, $e^{(1)}, \cdots, e^{(r)}$ are orthogonal central idempotents of $R_{0}$, summing to 1 .

For each $j$, we may identify the class of $K^{(j)}$-modules with the class of $R_{0}$-modules that are annihilated by all $e^{(k)}$ except $e^{(j)}$. Then every right (respectively left) $R_{0}$-module $M$ decomposes uniquely, as an $R_{0}$-module, into a 
direct sum of right (left) $K^{(j)}$-modules, $M=\bigoplus_{j} M e^{(j)}$ (resp. $M=\bigoplus_{j} e^{(j)} M$ ). We shall abbreviate $M e^{(j)}$ to $M^{j}$ ) (and $e^{(j)} M$ to ${ }^{(j} M$ ). Thus a right (left) $R_{0}$-module $M$ may be determined uniquely by specifying for each $j$ a right (left) $K^{(j)}$-module $M^{i}$ ) (resp. ${ }^{(j} M$ ).

If $R$ is an $R_{0}$-ring, the $e^{(j)}$ need not be central in $R$. If we decompose $R$ as a left module, $R=\bigoplus^{(j} R$, and then decompose each of the right ideals ${ }^{(j} R$ as a right $R_{0}$-module, we get a decomposition $R=\bigoplus_{j, k}{ }^{(j} R^{k)}$ into $\left(K^{(j)}\right.$, $\left.K^{(k)}\right)$-bimodules. Note that for $k \neq k^{\prime},{ }^{(j} R^{k)\left(k^{\prime}\right.} R^{l)}=0$ because $e^{(k)} e^{\left(k^{\prime}\right)}=$ $\{0\}$, while with $k=k^{\prime}$ we get ${ }^{(j} R^{k)(k} R^{l)} \subseteq\left({ }^{(j} R^{l)}\right.$. Intuitively, these formulas say that if we write an element of $R=\bigoplus^{\left(j R^{k}\right)}$ as an $r \times r$ matrix, putting the ${ }^{(j} R^{k)}$-summand in the $(j, k)$ th position, the multiplication of $R$ is consistent with the formalism of matrix multiplication. (But $R$ is not, in general, the full matrix ring over any ring $S$.)

Now assume all $K^{(j)}$ are sfields. The structure theory of $R_{0}$-modules is then hardly more complicated than that of vector-spaces over one sfield. An $R_{0}$ module $M$ is determined up to isomorphism by the $r$-tuple of cardinals $\left(\text { rank }_{K}^{(j)} M^{j)}\right)_{j=1}, \cdots, r$, and if we choose for each $M^{j)}$ a right $K^{(j)}$-basis $B^{j}$, then every element of $M$ may be written uniquely as a linear combination of the elements of these sets, where each $\left.b \in B^{j}\right)$ has coefficient in $K^{(j)}$, and almost all coefficients are zero. We shall call such an $r$-tuple of sets, $B=\left(B^{j}\right)$, a basis for the $R_{0}$-module $M$. (It is not, of course, a free basis.)

We see that every right module over $R_{0}$ is a direct sum of copies of the modules $K^{(j)}=e^{(j)} R_{0}$. Hence if $R_{\lambda}$ is an $R_{0}$-ring, the basic right $R_{\lambda}$-modules, as defined in $\S 1$, will be all direct sums of copies of the $R_{\lambda}$-modules $K^{(j)} \otimes_{R_{0}} R_{\lambda}$ $\cong{ }^{j} R_{\lambda} \quad(j=1, \cdots, r)$. Thus, if $U=\left(U^{j}\right)$ is an $r$-tuple of sets, we can form the "basic $R_{\lambda}$-module on the basis $U$ ":

$$
\bigoplus u^{\left(j_{R_{\lambda}}\right.} \quad\left(u \in U^{j)} ; u^{\left({ }^{j} R_{\lambda}\right.} \cong{ }^{(j} R_{\lambda} ; j=1, \cdots, r\right) .
$$

Let $\left(R_{\lambda}\right)_{\lambda \in \Lambda}$ be a family of faithful $R_{0}$-rings, let $R$ denote their coproduct over $R_{0}$, and for each $\mu \in \Lambda \cup\{0\}$, let $N_{\mu}$ be a (right) $R_{\mu}$-module.

For each $\mu$, and each $j=1, \cdots, r$, let us choose a $K^{(j)}$-basis $S_{\mu}^{j)}$ of $N_{\mu}^{j}$. For each $\lambda \in \Lambda$, and each $j, k=1, \cdots, r$, let us likewise choose a right $K^{(k)}$-basis of ${ }^{(j} R_{\lambda}^{k)}:{ }^{(j} T_{\lambda}^{k)}$ if $j \neq k$, or ${ }^{(j} T_{\lambda}^{j)} \cup\left\{e^{(j)}\right\}$ if $j=k$.

Let $S$ denote $\bigcup S_{\mu}^{j)}$, and $T$ denote $\bigcup^{(j} T_{\lambda}^{k)}$. For each $t \in{ }^{(j} T_{\lambda}^{k)}, j$ and $k$ will respectively be called the left and right indices of $t$, and $\lambda$ the $\Lambda$ index of $t$. A member of $S$ likewise has a right index, and, if it does not come from $N_{0}$, a $\Lambda$-index.

Let $U$ denote the set of all formal products $s t_{1} \cdots t_{n}$, where $n \geqslant 0$, $s \in S, t_{i} \in T$, no two successive terms have the same $\Lambda$-indices, but the right index of each term equals the left index of the next. We define the right index 
and the $\Lambda$-index of a member of $U$ as those of its last factor, and we partition the elements of $U$ by right index into subsets $U^{j)}(j=1, \cdots, r)$.

Following the development of $\S 4$, we form the $R_{0}$-module $N$ on the basis $\left(U^{j}\right)$ and give it a module structure over each $R_{\lambda}$, as the direct sum of $N_{\lambda}$ and a basic $R_{\lambda}$-module with basis $\left(U_{\sim \lambda}^{j)}\right)$. In particular, for each $u \in U_{\sim \mu}^{j)_{\mu}}$ we get an $R_{\mu}$-linear coordinate-map $c_{\mu u}: N \rightarrow{ }^{(j} R_{\mu}$. We verify that this $R$ module has the universal property of the standard $R$-module $N=\bigoplus N_{\mu} \otimes R$.

We well-order $S$ and $T$ arbitrarily, define a length-lexicographic ordering on $U$, and define degree, $\mu$-purity, leading term, etc. as in $\S 5$. The new right indices are ignored for these considerations. In Proposition 5.1 we must, of course, add the condition that the right index of each factor equals the left index of the next. Definition 5.2, of a well-positioned family, $\left(L_{\mu}\right)$ of $R_{\mu}$-submodules, goes over word for word.

Now let us call an element $x$ of a right $R_{0}$-module $M j$-homogeneous if $x \in M^{j)}$, equivalently, if $x e^{(j)}=x$, and homogeneous if it is $j$-homogeneous for some $j$. The key point in adapting the proofs of $\S \S 6-8$ to our new situation is to work with homogeneous elements. Note that if an element $y$ of any $R_{0}$ submodule $A \subseteq N$ has leading term $u$, say with $u \in U^{j}$, then the element $x=y e^{(j)}$ is $j$-homogeneous, and still belongs to $A$ and has leading term $u$; also that because it is $j$-homogeneous, we get $x R_{0} \cong{ }^{j} R_{0} \cong u R_{0}$. Similarly, if $u$ was the $\mu$-leading term of $y$, we see that $x R_{\mu} \cong{ }^{j} R_{\mu} \cong u R_{\mu}$.

So, for example, when we are setting the stage for a basic transfer in $\S 6$, if we take $x j$-homogeneous, then the surjective map $c_{\mu \nu} f: M \rightarrow{ }^{{ }^{j}} R_{\mu}$ is an isomorphism on $x R_{\mu}$, and so yields a decomposition $M_{\mu}=M_{\mu}^{\prime} \oplus x^{(j} R_{\mu}$.

Similarly, we choose the sets $Q_{\mu}$ and $Q_{0, \lambda}$ of $\S 8$ to consist of homogeneous elements with the desired key terms. They will then yield $R_{0}$-bases, in our new sense, for the submodules $L_{\mu}$.

The results of $\S \S 6-8$ thus go over, and hence Theorems 2.2 and 2.3 are true for this wider class of base-ring $R_{0}$.

10. Matrix rings (a special case of Morita equivalence). In contrast to the introduction of finite direct products in the preceding section, which was nontrivial, but straightforward, the introduction of matrix rings into these investigations is tedious but essentially trivial! (The main problem is where to write a new crop of superscripts.)

Let $K$ be any ring, and consider the $d \times d$ matrix ring over $K, \mathrm{~m}_{d}(K)$. We shall denote the matrix units, usually written $e_{p q}$, by $e^{q}(p, q=1$, $\cdots, d)$.

If $M$ is a right module over $\mathrm{m}_{d}(K)$ it has, in particular, a structụre of right module over the diagonal subring: 


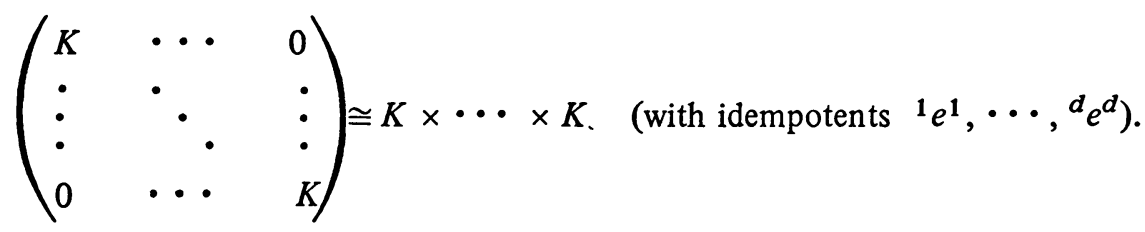

Hence $M$ can be decomposed into a direct sum of $d K$-modules, $\bigoplus_{p} M^{p}$. (We are using a slightly different notation from that of the preceding section $-M^{p}$ instead of $M^{j}$-to avoid conflict when we later combine the matrix and finite-direct-product constructions.) But in this case, multiplication by the nondiagonal matrix units establishes natural isomorphisms between these $K$-modules: $p_{e}^{q}: M^{p} \stackrel{\sim}{\rightarrow} M^{q}$. Hence $M=\bigoplus_{p} M^{1} e^{p}$. This direct sum can be represented as the module $\mathfrak{r}_{d}\left(M^{1}\right)$ of row vectors of length $d$ with entries in $M^{1}$, on which $\mathfrak{m}_{d}(K)$ acts by the usual conventions for multiplying a row vector and a matrix. Conversely, for any right $K$-module $M^{1}, \mathfrak{r}_{d}\left(M^{1}\right)$ gives us an $\mathfrak{m}_{d}(K)$ module. It is easy to check that every $\mathfrak{m}_{d}(K)$-module homomorphism $\mathfrak{r}_{d}\left(M^{\mathbf{1}}\right)$ $\rightarrow \mathfrak{r}_{d}\left(N^{1}\right)$ comes from a $K$-module homomorphism $M^{1} \rightarrow N^{1}$. Hence the correspondence $M=\mathfrak{r}_{d}\left(M^{1}\right) \leftrightarrow \leftrightarrow M^{1}$ gives us an equivalence of the category of right $\mathfrak{m}_{d}(K)$-modules with the category of right $K$-modules. (This equivalence, and the further equivalences to be described below, are special cases of the theory of Morita equivalence of rings. Cf. [7], [1, pp. 60-71].) Likewise, a left $\mathfrak{m}_{d}(K)$-module can be represented as a module of column vectors with entries in a left $K$-module, $M \cong \mathrm{c}_{d}\left({ }^{1} M\right)$; and given $K, K^{\prime}, d, d^{\prime}$, an $\left(\mathrm{m}_{d}(K), \mathrm{m}_{d^{\prime}}\left(K^{\prime}\right)\right)$ bimodule will look like the $d \times d^{\prime}$ matrices over the $\left(K, K^{\prime}\right)$-bimodule ${ }^{1} M^{1}$. In particular, we find that if $R$ is an $\mathrm{m}_{d}(K)$-ring, it will have not only as a bimodule but as a ring the structure $\mathrm{m}_{d}\left({ }^{1} R^{1}\right)$; and the expression of an $R$-module $M$ as a row-vector module over $\mathrm{m}_{d}(K): M=\mathfrak{r}_{d}\left(M^{1}\right)=\bigoplus M^{1} e^{p}$ will also be its decomposition as a row-vector module over $\mathrm{m}_{d}\left({ }^{1} R^{1}\right)=R$.

Thus, the theory of $m_{d}(K)$-rings and modules over them is functorially isomorphic to that of $K$-rings and their modules. Note, however, that under this correspondence, the free module of rank 1 over $K$ (or any $K$-ring) corresponds to an $\mathfrak{m}(K)$-module $\mathfrak{r}_{d}(K)$ which is not free; rather, the direct sum of $d$ copies of it is free of rank 1. (This is because the concept of a free module is not defined in terms of the structure of the category of modules alone, but also in terms of the "forgetful functor" | I: ModR $\rightarrow$ Set and our constructions do not respect this functor: $\left|\mathfrak{r}_{d}\left(M^{1}\right)\right| \cong\left|M^{1}\right|^{d}$.)

Now suppose we have rings $K^{(j)}$ and positive integers $d_{j}(j=1, \cdots, r)$. Let $R_{0}=\mathfrak{m}_{d_{1}}\left(K^{(1)}\right) \times \cdots \times \mathfrak{m}_{d_{r}}\left(K^{(r)}\right)$. If we combine the above observations about modules over matrix rings with our earlier ones about modules over finite direct products, we see that a right $R_{0}$-module $M$ can be written uniquely as $\left.\bigoplus_{j} \mathfrak{r}_{d_{j}}\left(M^{j}\right) 1\right)$. Hence there is a natural isomorphism between the category of 
such modules and that of $K^{(1)} \times \cdots \times K^{(r)}$-modules $\bigoplus_{j} M^{j) 1}$. One obtains the former module from the latter by "repeating the $j$ th factor $d_{j}$ times," for each $j$.

We likewise find that, if we represent a $K^{(1)} \times \cdots \times K^{(r)}$-ring $R$ as a matrix of bimodules ${ }^{(j} R^{k)}$ (with appropriate multiplications defined between them), then the most general $R_{0}$-ring can be obtained from such an $R$ by replacing each ${ }^{(j} R^{k)}$ by a $d_{j} \times d_{k}$ block of copies of itself. Hence the theory of $R_{0}$-rings and modules over these is functorially isomorphic to that of $K^{(1)} \times$ $\cdots \times K^{(r)}$-rings and their modules.

But the definition of a coproduct is category-theoretic! Hence to construct a coproduct of $R_{0}$-rings, we can find the corresponding $K^{(1)} \times \cdots \times K^{(r)}$. rings, form their coproduct in the category of such rings, then go back up to the associated $R_{0}$-ring. The statements of Proposition 2.1 and Theorems 2.2 and 2.3 are also category-theoretic, hence they will go over from the case $R_{0}$ a direct product of skew fields, $K^{(1)} \times \cdots \times K^{(r)}$, to $R_{0}$ a product of full matrix rings over skew fields.

(One can, of course, alternatively, follow the approach of the preceding section and extend the methods of $\S \S 4-8$ to cover this case. But the present method is far more pleasant.)

\section{Bound and quasifinite modules, arbitrary modules; chain conditions.} Note that in the proof of Lemma 6.1, the hypothesis that $M$ is finitely generated-equivalently, that all $M_{\mu}$ are finitely generated and almost all are zerowas used only to prove that the image-modules $f\left(M_{\mu}\right)$ each have finite $\mu$-supports, almost all of which are empty. Suppose we call a module $M$ over a ring $R$ bound if $\operatorname{Hom}(M, R)=\{0\}$ (the usage is due to Cohn), and quasifinite if every homomorphic image of $M$ in a free module $F$ lies in a finitely generated submodule of $F$. (E.g., any right ideal of a right Ore ring is quasifinite; so is any extension of a bound module by a finitely generated module.) It follows from Proposition 2.1 that a standard module $\bigoplus M_{\mu} \otimes R$ over our coproduct ring $R_{\mu}$ is bound if and only if each $M_{\mu}$ is a bound $R_{\mu}$-module, and quasifinite if and only if all the $M_{\mu}$ are quasifinite and almost all are bound.

Clearly, the proof of Lemma 6.1 works with $M$ assumed quasifinite rather than finitely generated. Hence we can also weaken "finitely generated" to "quasifinite" in Proposition 6.2, Theorem 2.3, and Corollaries 2.8-2.11.

I wonder whether any modification of these results can be obtained for arbitrary standard modules. The proof of Proposition 6.2 suggests that an analog might hold for nonfinitely generated modules, in which the isomorphism $\alpha$ is replaced by some sort of topological limit of finite compositions of transvections and basic transfers, such that only finitely many affect any given element of $M$. 
(But will this limit be an isomorphism?) To conjecture a plausible analog of Corollary 2.8, note that if $\kappa$ is any infinite cardinal, and $\operatorname{Mod}^{\kappa} R$ the category of right $R$-modules generated by less than $\kappa$ elements, then the isomorphism classes of objects of $\operatorname{Mod}^{\kappa} R$ form an abelian "k-semigroup". $S_{\oplus}^{\kappa}\left(\operatorname{Mod}^{\kappa} R\right)$, that is, a semigroup with commutative associative addition defined for all families of fewer than $\kappa$ elements. Conceivably, $S_{\oplus}^{\kappa}\left(S t d^{\kappa} R\right)$ might be the pushout of the $\kappa$-semigroups $S_{\oplus}^{\kappa}\left(\operatorname{Mod}^{\kappa} R_{\lambda}\right)$ over $S_{\oplus}^{\kappa}\left(\operatorname{Mod}^{\kappa} R_{0}\right)$.

I have said nothing so far about chain conditions in coproduct rings. Of course, these rings are generally non-Noetherian; e.g., the coproduct over a field $k$ of two copies of the Noetherian polynomial algebra $k[x]$ is the free associative algebra on two indeterminates, $k\left\langle x_{1}, x_{2}\right\rangle$, which is very non-Noetherian. But this ring (and in fact any fir) does satisfy more subtle conditions: for each positive integer $n, R$ has ACC on right ideals generated by $\leqslant n$ elements, and for every right ideal $J, R$ has ACC on right ideals $I \supseteq J$ such that $I / J$ is bound as a right module [16, Theorems 1.2.3 and 5.8.2]. The former condition is also satisfied by any ring $R$ with $n$-term weak algorithm ([15, Theorem $2.4]$, but not by all $n$-firs $[15, \S 4])$. These conditions have proved of considerable value in the study of such rings (cf. [16, Chapter 6], [5]). Hence it would be worth knowing whether such conditions are respected by coproducts. I suspect that the methods of the preceding sections can be used to prove such results. Given a chain $I_{1} \subseteq I_{2} \subseteq \cdots$ of right ideals of $R$ (or of submodules of an appropriate standard $R$-module), let $\left(L_{i \mu}\right)$ denote the well-positioned system of $R_{\mu}$-submodules generating $I_{i}(i=1,2, \cdots)$, constructed as in the proof of Proposition 7.1. By studying these systems one should be able to obtain conditions for this chain to stabilize, in terms of the module-theory of the $R_{\mu}$. But I have not examined this question carefully. Cf. also [25].

12. A class of simple examples: coproducts of two quadratic extensions. It is well-known that the one case in which a nontrivial coproduct of groups is "reasonably" small is that of two copies of $\mathbf{Z}_{2}$. This coproduct, defined by two generators $a, b$ and two relations $a^{2}=e, b^{2}=e$, is the infinite dihedral group.

It is likewise easy to see from the normal form results of $\S 4$ applied to $R$ itself that for $R_{0}$ a sfield, the one nontrivial case in which our right basis for the coproduct ring $R$ will be reasonably small is when $\Lambda$ consists of exactly two elements, and each $R_{\lambda}$ is 2-dimensional over $R_{0}$. We shall examine a few such cases in this section. As we observed at the end of $\S 4$, the normal form results obtained there really require less than that $R_{0}$ be a sfield. In the following examples, the base ring $R_{0}$ will always be commutative, but not necessarily a field. 
Our first example has bearing on the global dimension question to be discussed in the next section. Otherwise, the results of this section are not very relevant to the rest of the paper.

For more on the coproduct of two quadratic sfield extensions of a sfield $R_{0}$, see Cohn [10, $\left.\S 7,8\right]$, and for certain other cases, Smits [21], [29].

EXAMPLE 12.1. The coproduct over the integers of two copies of the Gaussian integers, $\mathbf{Z}[i]$. This ring is generated by two elements, $i$ and $i^{\prime}$, with defining relations $i^{2}=-1, i^{\prime 2}=-1$. Let us take new generators $i$ and $x=$ $i i^{\prime}$. Then the second defining relation becomes $(-i) x(-i) x=-1$. Hence $x$ is invertible in $R$, and $R$ can in fact be described as the result of adjoining to $P=\mathrm{Z}\left[x, x^{-1}\right]$ an element $i$ with square -1 , satisfying $i x=x^{-1} i$. (Note. This is not an "anticommutativity relation" $x y=-y x$.) Hence $i A=A i$ for all $A \in P$, where ${ }^{-}$is the automorphism of $P$ sending $x$ to $x^{-1}$.

Let $M$ be any $P$-module. From the fact that $R$ is projective as left and right $P$-module (in fact, it is free on the basis $\{1, i\}$ ) and contains $P$ as a direct summand of $P$-bimodules, we can deduce that $\mathrm{hd}_{R} M \otimes R \geqslant \mathrm{hd}_{P} M \otimes R$ $=\mathrm{hd}_{P} M$, hence $\mathrm{r} \mathrm{gl} \operatorname{dim} R \geqslant \mathrm{r} g l \operatorname{dim} P$. But the commutative ring $P=$ $\mathrm{Z}\left[x, x^{-1}\right]$ is known to have global dimension $2[12, \mathrm{p} .174$, Theorem 6 and following exercise], so $\mathrm{r} g l \operatorname{dim} R \geqslant 2$, though $\mathrm{r} g l \operatorname{dim} Z[i]=\mathrm{r} \operatorname{gl} \operatorname{dim} Z\left[i^{\prime}\right]$ $=\mathrm{r} g \mathbf{g} \operatorname{dim} \mathbf{Z}=1$.

Further remarks: If we tensor this ring with the field $\mathbf{Q}$ of rationals, the resulting ring $R \otimes \mathbf{Q}$ will be the coproduct over $\mathbf{Q}$ of two copies of $\mathbf{Q}(i)$, hence by Corollary 2.5 , right hereditary. In fact, by $[10, \S 7$, Lemma 2] it is a principal right and left ideal domain.

Note that the center of $R$ is $\mathrm{Z}[t]$, where $t=x+x^{-1}$, and that $R$ is free of rank 4 over this subring. It is not hard to show that given relatively prime elements $A, B \in P$, we have $(A+B i) R \cap \mathbf{Z}[t]=(A \bar{A}+B \bar{B}) \mathbf{Z}[t]$. Thus the map $A+B i \mapsto(A+B i)(\bar{A}-i \bar{B})=A \bar{A}+B \bar{B}$ is a kind of "quaternionic norm" of $R$ into its center.

12.2. Centers. In fact, the structure of the center in the above example is typical of a wide class of cases. Let $C$ be any commutative ring, and $C[\alpha]$, $C[\beta]$ two-dimensional $C$-algebras, defined by quadratic equations $\alpha^{2}-a \alpha-a^{\prime}$ $=0$ and $\beta^{2}-b \beta-b^{\prime}=0\left(a, a^{\prime}, b, b^{\prime} \in C\right)$, and let us abbreviate $a-\alpha=$ $\bar{\alpha}, b-\beta=\bar{\beta}$. The coproduct ring $R$ of $C[\alpha]$ and $C[\beta]$ over $C$ will, by the results of $\S 4$, be free as a $C$-module on the basis $\left\{(\alpha \beta)^{n}, \beta(\alpha \beta)^{n},(\alpha \beta)^{n} \alpha\right.$, $\beta(\alpha \beta)^{n} \alpha \mid n \geqslant 0$ \}. Now consider the element $t=\alpha \beta+(b-\beta)(a-\alpha)=\alpha \beta+$ $\bar{\beta} \bar{\alpha}$ of $R$. $t$ is central in $R$. (It suffices to show it commutes with $\alpha$ and $\beta$, and by symmetry, it is enough to consider $\alpha$. Expanding $t$ in $\alpha$ and $\beta$, and using the formula $[\alpha, \alpha \beta+\beta \alpha]=\left[\alpha^{2}, \beta\right]$, one arrives at $[\alpha, t]=$ $\left[\alpha^{2}-a \alpha, \beta\right]=\left[a^{\prime}, \beta\right]=0$.) It is also not hard to calculate in terms of our 
normal form that if $s$ is any element of positive degree in the center of $R$, then the highest-degree component of $s$ must have the form $c(\alpha \beta)^{n}+c(\beta \alpha)^{n}$ $(c \in C)$, which is the leading term of $c t^{n}$. We deduce that the center of $R$ is the polynomial ring $C[t]$, as in the preceding example. Easy calculations show that $R$ is free as a $C[t]$-module on $\{1, \alpha, \beta, \alpha \beta\}$. In particular, it is free on $\{1, \alpha\}$ as a right module over the commutative subring $C[\beta, t]$, and this yields a representation by $2 \times 2$ matrices over this ring:

$$
\alpha \mapsto\left(\begin{array}{rr}
0 & -a^{\prime} \\
1 & a
\end{array}\right), \quad \beta \mapsto\left(\begin{array}{cc}
\beta & t-a \bar{\beta} \\
0 & \bar{\beta}
\end{array}\right) \quad \text { and }\left(t \mapsto\left(\begin{array}{ll}
t & 0 \\
0 & t
\end{array}\right)\right) \text {. }
$$

(Cf. $[21, \S 6]$.

The trace and norm of this representation carry $R$ into $C[t]$, and are equal to $A \mapsto A+\bar{A}$ and $A \mapsto A \bar{A}$, where " $"$ is the involution of $R$ induced by the involutions "-" of $C[\alpha]$ and $C[\beta]$.

(Except in the case of two quadratic extensions, the center of a coproduct ring generally lies in the center of the base-ring, $R_{0}$. Exercise. When $R_{0}$ is a field, prove that a nontrivial coproduct of $R_{0}$-algebras has center precisely $R_{0}$, except in the two-quadratics case.)

12.3. Idempotents and nilpotents (split quadratic extensions). For the study of idempotent and nilpotent elements in an arbitrary $C$-algebra, it could be useful to know the structures of coproducts of copies of the algebras $C[\iota]\left(\iota^{2}=\imath\right)$ $\cong C \times C$, and $C[\nu]\left(\nu^{2}=0\right)$. Let $C[\alpha]$ and $C[\beta]$ be any pair of these algebras. The results of the above subsection apply to their coproduct $R$; in particular, we get the matrix representation (3) over $C[\beta, t]$. But here note that there exists an augmentation $C[\beta] \rightarrow C$ (taking $\beta$ to 0 ). Hence from the representation (3) we get a representation by $2 \times 2$ matrices over $C[t]$. Surprisingly, this turns out to be faithful in these cases. We can furthermore easily write down the resulting subalgebra of $\mathfrak{m}_{2}(C[t])$. The results are listed below. (We have made one modification: when $\alpha=\imath$, we have applied a similarity transformation to bring the representing matrix to the form $\left(\begin{array}{ll}0 & 0 \\ 0 & 1\end{array}\right)$ rather than $\left(\begin{array}{ll}0 & 0 \\ 1 & 1\end{array}\right)$.) $)$ To verify that each of the representations below is faithful, simply note that the algebra of matrices involved is free of rank 4 as a $C[t]$-module!

Throughout, $E, F, G$ and $H$ will denote elements of $C[t]$.

(i) Two idempotents, $\alpha=\imath, \beta=i$. (This calculation was suggested and carried out with the author by Alan G. Waterman, and in turn suggested the other cases.)

$$
R=\left\{\left(\begin{array}{cc}
E & t F \\
(1-t) G & H
\end{array}\right)\right\}, \quad \iota=\left(\begin{array}{ll}
0 & 0 \\
0 & 1
\end{array}\right), \quad i^{\prime}=\left(\begin{array}{cc}
1-t & t \\
1-t & t
\end{array}\right)
$$


(ii) An idempotent $\iota$ and a nilpotent $\nu$. Here, depending on which we choose to be " $\alpha$ ", we get a representation putting $\iota$ or $\nu$ in simpler form:

$$
R=\left\{\left(\begin{array}{cc}
E & t F \\
t G & H
\end{array}\right)\right\}, \quad \iota=\left(\begin{array}{ll}
0 & 0 \\
0 & 1
\end{array}\right), \nu=\left(\begin{array}{cc}
-t & t \\
-t & t
\end{array}\right),
$$

or

$$
R=\left\{\left(\begin{array}{cc}
E & t E-t G+t^{2} F \\
G & H
\end{array}\right)\right\}, \quad \iota=\left(\begin{array}{ll}
1 & t \\
0 & 0
\end{array}\right), \nu=\left(\begin{array}{ll}
0 & 0 \\
1 & 0
\end{array}\right) .
$$

(iii) Two nilpotents.

$$
R=\left\{\left(\begin{array}{cc}
E & t F \\
G & E+t H
\end{array}\right)\right\}, \quad \nu=\left(\begin{array}{ll}
0 & 0 \\
1 & 0
\end{array}\right), \nu^{\prime}=\left(\begin{array}{ll}
0 & t \\
0 & 0
\end{array}\right) .
$$

Finally, an example with noncentral $R_{0}$ :

EXAMPLE 12.4. The coproduct over the complex numbers of two copies of the quaternions. By Corollary 2.12 this ring will be a fir, and in fact by $[10, \S 7$, Lemma 2], it is a principal right and left ideal domain.

$R$ will be generated over the complexes, C, by two elements $j$ and $j^{\prime}$, each commuting with the real numbers, $\mathrm{R}$, anticommuting with $i$, and having square -1 . Let us take $j$ and $x=i j^{\prime}$ for new generators of $R$ over $\mathrm{C}$. The latter element will commute with $i$, since $j$ and $j$ both anticommute with it; the defining relation $j^{\prime 2}=-1$ takes the form $j x=x^{-1} j$.

Now the automorphism induced by conjugation by $j$ on the commutative subring $\mathbf{C}\left[x, x^{-1}\right]$ has the same form as complex conjugacy on the function ring $\mathrm{C}\left[e^{i \theta}, e^{-i \theta}\right]$ for $\theta$ a real variable; so let us formally rename $x$ as $e^{i \theta}$. But we also know that $\mathbf{C}\left[e^{i \theta}, e^{-i \theta}\right]$ is generated over $\mathbf{C}$ by the real functions $\cos \theta=\left(e^{i \theta}+e^{-i \theta}\right) / 2$ and $\sin \theta=\left(e^{i \theta}-e^{-i \theta}\right) / 2 i$. So:

$$
R \cong \mathbf{C}\left[e^{i \theta}, e^{-i \theta}\right][j]=\mathbf{R}[\sin \theta, \cos \theta][i, j] \cong \mathbf{R}[\sin \theta, \cos \theta] \otimes \mathbf{R}(i, j),
$$

i.e., our ring $R$ is the tensor product over the reals of the trigonometric function ring, and the quaternions. Note that the center of $R, \mathbf{R}[\sin \theta, \cos \theta]$, unlike$R$ itself, is not a principal ideal domain. But it is a Dedekind domain (hereditary commutative integral domain). In fact, Robson and Small have recently shown that the center of a right hereditary prime ring with polynomial identity is always a Dedekind domain [20].

13. The global dimension formula, and related questions. In the main results of this paper, we took for our base ring $R_{0}$ any ring of global dimension zero ( $=$ finite direct product of matrix rings over skew fields). Example 12.1 showed that the formula for the global dimension of a coproduct (Corollary 2.5) 
fails for $R_{0}=\mathrm{Z}$, a ring of global dimension 1. The way in which it failed suggests that a generalization of the formula might be:

$$
\mathrm{r} g l \operatorname{dim} R \leqslant \sup \left(1+\mathrm{rgl} \operatorname{dim} R_{0} ; \mathrm{rgl} \operatorname{dim} R_{\lambda}(\lambda \in \Lambda)\right) .
$$

AdDED IN PRoof (June 12, 1974). I have just learned that this and related results for group rings have been known to topologists for several years $([30, \S 2]$ and [31, p. 176], cf. [27], [28, Propositions 7, 8]) and that they have been aware that the same methods are applicable to more general ring extensions $R_{0} \subseteq$ $R_{\lambda}(\lambda \in \Lambda)$ as long as all $R_{\lambda}$ have good left and right $R_{0}$-module structurese.g., $R_{\lambda} / R_{0}$ flat on one side and projective on the other [6]. So it seems that the "open question" answered by Corollary 2.5 was "open" only among ringtheorists!

ADDED IN Proof (September 1, 1974). Recently, Warren Dicks (Bedford College, London) has obtained by simple methods some beautiful homological results on colimits of trees of rings, which in particular yield (4) and the corresponding inequality for weak global dimension under only the assumption that $R$ be left-flat over every $R_{\mu}$ [26, Corollary 7]! (END of ADDED MATERIAL.)

On the other hand, Cohn [14, Introduction] suggests that results as good as those holding over sfields (e.g., analogs of all results in $\$ 2$ above?) may hold for coproducts over general rings $R_{0}$ if all $R_{\lambda}$ satisfy some kind of generalized "inertia" conditions over $R_{0}$. (These would be conditions saying that if an element or matrix $A$ over $R_{0}$ factors over $R_{\lambda}$, this factorization reduces in some way to a factorization of $A$ over $R_{0}$. Thus, the equation $2=(1+i)$. $(1-i)$ is an example of the noninertness of $\mathbf{Z}$ in $\mathbf{Z}[i]$.)

It would also be interesting to see whether coproducts over a ring $R_{0}$ with weak global dimension 0 , i.e., a von Neumann regular ring, can be proved to have particularly good properties.

14. Coproducts of nonfaithful $R_{0}$-rings. In $\S \S 2-10$, we assumed that all $R_{\lambda}$ were faithful $R_{0}$-rings. To examing the nonfaithful case, consider a ring $R_{0}$ and a family of $R_{0}$-rings $\left(R_{\lambda}\right)$. Call a 2-sided ideal $I \subseteq R_{0} \lambda$-stable, for a given $\lambda \in \Lambda$, if it is the inverse image of some 2-sided ideal of $R_{\lambda}$, that is, if $R_{\lambda} / R_{\lambda} I R_{\lambda}$ is a faithful $R_{0} / I$-ring. If $R_{\lambda}$ is not itself a faithful $R_{0}$-ring, $\{0\} \subseteq$ $R_{0}$ will not be $\lambda$-stable. However since the class of $\lambda$-stable ideals is closed under intersections, there will exist a unique minimal ideal $I \subseteq R_{0}$ which is $\lambda$-stable for all $\lambda \in \Lambda$. This ideal $I$ must go to zero in the coproduct $R$ of the $R_{0}$-rings $R_{\lambda}$; hence $R$ can be considered the coproduct over $R_{0}^{\prime}=R_{0} / I$ of the rings $R_{\lambda}^{\prime}=R_{\lambda} / R_{\lambda} I R_{\lambda}$, and these are all faithful $R_{0}^{\prime}$-rings.

Thus, the study of coproducts of arbitrary rings over a given ring $R_{0}$ can be reduced to a combination of the study of copriducts of faithful ring-extensions, 
such as we have pursued here, and the study of the quotients $R_{\lambda}^{\prime}$ of an $R_{0}$-ring $R_{\lambda}$ by a "basic" ideal, i.e., one of the form $R_{\lambda} I R_{\lambda}\left(I \subseteq R_{0}\right)$. Note that the quotient ring $R_{\lambda}^{\prime}=R_{\lambda} / R_{\lambda} I R_{\lambda}$ can be described as the coproduct over $R_{0}$ of the two rings $R_{\lambda}$ and $R_{0} / I$.

What can be said about such quotients $R^{\prime}=R / R I R\left(I \subseteq R_{0}\right)$ in the case where $R_{0}$ is a ring of global dimension 0 ? (We henceforth suppress the " $\lambda$ ".) By analogy with the results of $\S 2$, we would hope that "good" properties of $R$ would be retained, e.g., that $\mathrm{r} g \mathrm{dim} R^{\prime} \leqslant \mathrm{r} \mathrm{gl} \operatorname{dim} R$. In [3, §11] we shall see that, using Morita equivalence, the question becomes essentially equivalent to that of what good properties of a ring $R$ are preserved when we divide out by the trace ideal of any finitely generated projective module. It appears that this construction is not as benign as that of coproducts of faithful $R_{0}$-rings. E.g., the analog of Corollary 2.6 is false. But our knowledge of the behavior of this construction is quite fragmentary.

For reasons also explained in $[3, \S 10]$, if we had results abijut the coproduct over a triangular matrix ring, $R_{0}=\left(\begin{array}{cc}K & K \\ 0 & K\end{array}\right)$ ( $K$ a sfield), of a faithful $R_{0}$-ring $R$ with the full matrix ring $\mathrm{m}_{2}(K)$, this would give us information about the construction of adjoining to a ring the inverse of an arbitrary nonzero map between finitely generated projective modules, and in particular, the process of adjoining the inverse of an element.

15. A reduction to category theory? The techniques of $\S \S 1-10$ involved us in the study of modules over our coproduct ring $R$, but hardly ever required us to look at the ring $R$ itself.

Now from the point of view of the theory of algebraic structures, if $R$ is a coproduct as in $\S 1$, then the theory of $R$-modules, in the sense of [18], will be the coproduct over the theory of $R_{0}$-modules of the theories of $R_{\lambda}$-modules $(\lambda \in \Lambda$ ). (Or their product, depending on which way one considers morphisms of algebraic theories to run.) Suppose, generally, that we are given a family of algebraic theories $T_{\lambda}$ over a common theory $T_{0}$, and we form their coproduct $T$. Let Set $^{T_{0}}$ etc. denote the categories of models of these theories. Can one directly construct the category $\operatorname{Set}^{T}$ from the categories $S e t^{T_{\mu}}$ and the given functors connecting them? If so, and if this category-theoretic construction has a reasonable form, one might try to obtain the present results from more general results about this construction applied to appropriate sorts of additive categories.

(Cf. [24], where for any family $\left(T_{\lambda}\right)$ of theories, it is shown how to construct the category $\operatorname{Set}^{\Sigma T_{\lambda}}$ from the categories $\operatorname{Set}^{T_{\lambda}}$. Here $\Sigma$ denotes the "symmetric product" operation on theories. When $T_{\lambda}$ is the theory of $R_{\lambda}$ " modules for each $\lambda \in \Lambda, \Sigma T_{\lambda}$ is the theory of $\otimes_{Z} R_{\lambda}$-modules.) 
In any case, it might be of interest to study category-theoretic analogs of the situation of this paper. E.g., given small abelian categories $C_{0},\left(C_{\lambda}\right)$, and faithful right exact functors $\phi_{\lambda}: C_{0} \rightarrow C_{\lambda}$, will this family have a pushout $C$ in the category of small abelian categories and right exact functors? If so, what can be said of the homological properties of this pushout C?

In $[3, \S 12]$ a different sort of category-theoretic generalization is suggested. In particular, it is clear from the discussion there that there is essentially no difference between constructions with rings and constructions with their additive categories of finitely generated projective modules.

\section{REFERENCES}

1. H. Bass, Algebraic K-theory, Benjamin, New York, 1968. MR 40 \#2736.

2. G. M. Bergman, Hereditarily and cohereditarily projective modules, Ring Theory (Proc. Sympos., Park City, March 1971), Academic Press, New York, 1972, pp. 20-62.

3. Coproducts and some universal ring contructions, Trans. Amer. Math. Soc. 200 (1974), 33-87.

4. The diamond lemma in ring theory (to appear).

5. G. M. Bergman and P. M. Cohn, The centres of 2-firs and hereditary rings, Proc. London Math. Soc. (3) 23 (1971), 83-98. MR 45 \#294.

6. Sylvain Cappell, (personal communication).

7. P. M. Cohn, Morita equivalence and duality, Queen Mary College Mathematics Notes, Queen Mary College, London, 1969. MR 41 \#3530.

8. - On the free product of associative rings. I, Math. Z. 71 (1959), 380-

398. MR $21 \# 5648$.

9. - On the free product of associative rings. II. The case of (skew) fields, Math. Z. 73 (1960), 433-456. MR 22 \#747.

10. - Quadratic extension of skew fields, Proc. London Math. Soc. (3) 11 (1961), 531-556. MR 25 \#101.

11. On a generalization of the Euclidean algorithm, Proc. Cambridge Philos. Soc. 57 (1961), 18-30. MR 22 \#9514; errata, 22, 2547.

12. - Rings with weak algorithm, Trans. Amer. Math. Soc. 109 (1963), 332 -

356. MR 27 \#3657.

13. Universal algebra, Harper and Row, New York, 1965. MR 31 \#224.

14. - On the free product of associative rings. III, J. Algebra 8 (1968),

376-383; correction, ibid. 10 (1968), 123. MR 36 \#5170; 37 \#4121.

15. - Dependence in rings. II. The dependence number, Trans. Amer. Math. Soc. 135 (1969), 267-279. MR 43 \#4848.

16. - Free rings and their relations, Academic Press, New York, 1971.

17. - Euclid's elements, 2nd ed., Dover, New York, 1956. MR 17, 814.

18. Peter Freyd, Algebra valued functors in general and tensor products in particular, Colloq. Math. 14 (1966), 89-106. MR 33 \#116.

19. I. Kaplansky, Fields and rings, Univ. of Chicago Press, Chicago, Ill., 1969. MR 42 \#345.

20. J. C. Robson and Lance W. Small, Hereditary prime P. I. rings are classical hereditary orders, J. London Math. Soc. (to appear). 
21. T. H. M. Smits, The free product of a quadratic number field and a semi-field, Nederl. Akad. Wetensch. Proc. Ser. A 72 = Indag. Math. 31 (1969), 145-159. MR 39 \#5631.

22. J. R. Stallings, Whitehead torsion of free products, Ann. of Math. (2) 82 (1965), 354-363. MR 31 \#3518.

23. S. Stevin, Arithmétique (1585) (vol. Il of collected works, 1958).

24. A. G. Waterman, General-valued polarities, Doctoral Thesis, Harvard University, Cambridge, Mass., 1971.

25. K. G. Choo, K. Y. Lam and E. Luft, On free products of rings and the coherence property, in Algebraic $K$-theory. II: "Classical" algebraic $K$-theory and connections with arithmetic, H. Bass (editor), Lecture Notes in Math., vol. 342, Springer-Verlag, Berlin, 1973, pp. 135-143.

26. W. Dicks, Mayer-Vietoris presentations over coproducts of rings (to appear).

27. R. Lyndon, Cohomology theory of groups with a single defining relation, Ann. of Math. (2) 52 (1950), 650-665. MR 13, 819.

28. J.-P. Serre, Cohomologie des groupes discrètes, in Prospects in Mathematics, Ann. of Math. Studies, no. 70, Princeton Univ. Press, Princeton, N. J., 1971, pp. 77-170.

29. T. H. M. Smits, On special hereditary orders in quaternion algebras, Delft Progress Report (Delft U. Of Techn., the Netherlands) (F), 1 (1974), 37-40.

30. R. Swan, Groups of cohomological dimension one, J. Algebra 12 (1969), 585610. MR 39 \#1521.

31. F. Waldhausen, "Whitehead groups of generalized free products", in Algebraic $K$-theory. II: "Classical" algebraic $K$-theory and connections with arithmetic, H. Bass (editor). Lecture Notes in Math., vol. 342, Springer-Verlag, Berlin, 1973, pp. 155-182.

DEPARTMENT OF MATHEMATICS, UNIVERSITY OF CALIFORNIA, BERKELEY, CALIFORNIA 94720 